\title{
Measurement of the hadronic activity in events with a $Z$ and two jets and extraction of the cross section for the electroweak production of a $Z$ with two jets in pp collisions at $\sqrt{s}=7 \mathrm{TeV}$
}

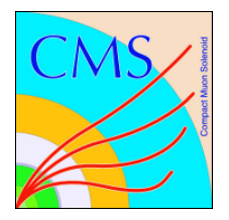

\section{The CMS collaboration}

\section{E-mail: cms-publication-committee-chair@cern.ch}

ABSTRACT: The first measurement of the electroweak production cross section of a $\mathrm{Z}$ boson with two jets (Zjj) in pp collisions at $\sqrt{s}=7 \mathrm{TeV}$ is presented, based on a data sample recorded by the CMS experiment at the LHC with an integrated luminosity of $5 \mathrm{fb}^{-1}$. The cross section is measured for the $\ell \ell \mathrm{jj}(\ell=\mathrm{e}, \mu)$ final state in the kinematic region $m_{\ell \ell}>50 \mathrm{GeV}, m_{\mathrm{jj}}>120 \mathrm{GeV}$, transverse momenta $p_{\mathrm{T}}^{\mathrm{j}}>25 \mathrm{GeV}$ and pseudorapidity $\left|\eta^{\mathrm{j}}\right|<4$.0. The measurement, combining the muon and electron channels, yields $\sigma=154 \pm$ 24 (stat.) \pm 46 (exp. syst.) \pm 27 (th. syst.) \pm 3 (lum.) fb, in agreement with the theoretical cross section. The hadronic activity, in the rapidity interval between the jets, is also measured. These results establish an important foundation for the more general study of vector boson fusion processes, of relevance for Higgs boson searches and for measurements of electroweak gauge couplings and vector boson scattering.

KEYwords: Hadron-Hadron Scattering

ArXiv EPRINT: 1305.7389 


\section{Contents}

1 Introduction 1

2 CMS detector, reconstruction, and event simulation 3

3 Event selection $\quad 5$

4 Hadronic activity in the rapidity interval between tagging jets $\quad 9$

$\begin{array}{lll}4.1 & \text { Central hadronic activity measurement using jets } & 10\end{array}$

$\begin{array}{ll}\text { 4.2 Central hadronic activity measurement with track jets } & 11\end{array}$

5 Measurements of the radiation patterns in multijet events in association $\begin{array}{ll}\text { with a } \mathrm{Z} \text { boson } & \mathbf{1 2}\end{array}$

6 Signal cross section measurement 13

6.1 Signal extraction using the dijet mass fit 13

$\begin{array}{lll}6.2 & \text { Systematic uncertainties } & 15\end{array}$

$\begin{array}{lll}\text { 6.3 Signal extraction using MVA analysis } & 17\end{array}$

$\begin{array}{lll}6.4 \text { Results } & 20\end{array}$

$\begin{array}{lll}7 & \text { Summary } & 22\end{array}$

$\begin{array}{lr}\text { The CMS collaboration } & 26\end{array}$

\section{Introduction}

The cross section for the electroweak (EW) production of a central $\mathrm{W}$ or $\mathrm{Z}$ boson in association with two jets that are well separated in rapidity is quite sizable at the Large Hadron Collider (LHC) [1]. These electroweak processes have been studied in the context of rapidity intervals in hadron collisions [2,3], as a probe of anomalous triple-gauge-boson couplings [4], and as a background to Higgs boson searches in Vector Boson Fusion (VBF) processes [5-8]. There are three classes of diagrams to be considered in the EW production of $\mathrm{W}$ and $\mathrm{Z}$ bosons with two jets: VBF processes, bremsstrahlung, and multiperipheral processes. A full calculation reveals a large negative interference between the pure VBF process and the other two categories [1,3]. Figure 1 shows representative Feynman diagrams for these EW $\ell$ lqq' production processes. A representative Feynman diagram for Drell-Yan production in association with two jets is shown in figure 2. This process is the dominant background in the extraction of EW $\ell \ell q^{\prime}$ cross section. In what follows we designate as "tagging jets" the jets that originate from the fragmentation of the outgoing quarks in the EW processes shown in figure 1. 

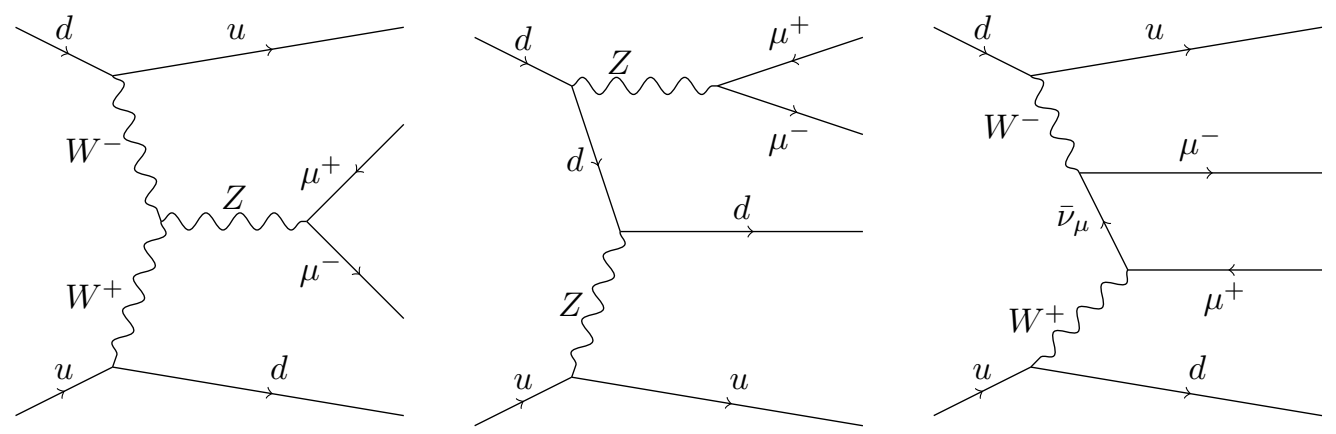

Figure 1. Representative diagrams for EW $\ell \ell q q^{\prime}$ production (for $\ell=\mu$ ): VBF (left), bremsstrahlung (middle), and multiperipheral (right).

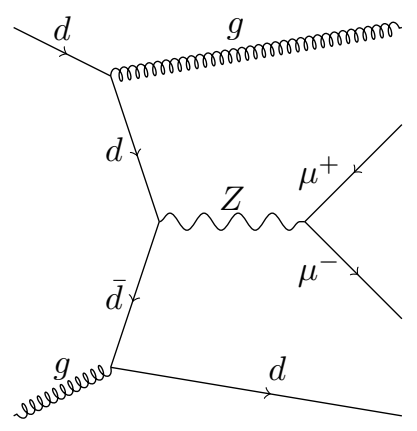

Figure 2. Representative diagram for Drell-Yan production in association with two jets.

The study of these processes establishes an important foundation for the more general study of vector boson fusion processes, of relevance for Higgs boson searches and for measurements of electroweak gauge couplings and vector-boson scattering. The VBF Higgs boson production in proton-proton (pp) collisions at the LHC has been extensively investigated $[9,10]$ as a way to discover the particle and measure its couplings [11-13]. Recent searches by the ATLAS and CMS collaborations for the standard model (SM) Higgs boson include analyses of the VBF final states [14, 15].

In particular, the study of the processes shown in figure 1 can improve our understanding of the selection of tagging jets as well as that of vetoing additional parton radiation between forward-backward jets in VBF searches [5-8]. The measurement of the electroweak production of the $\mathrm{Zjj}$ final state is also a precursor to the measurement of elastic vector boson pair scattering at high energy, an important physics goal for future analyses of LHC data.

In this work we measure the cross section for electroweak $\mathrm{Z}$ boson production in association with two jets in pp collisions at a center-of-mass energy of $7 \mathrm{TeV}$, where the $\mathrm{Z}$ boson decays into $\mu^{+} \mu^{-}$or $\mathrm{e}^{+} \mathrm{e}^{-}$, using a data sample collected in 2011 by the Compact Muon Solenoid (CMS) experiment with an integrated luminosity of $5.1 \mathrm{fb}^{-1}$ for the $\mu^{+} \mu^{-}$mode and $5.0 \mathrm{fb}^{-1}$ for the $\mathrm{e}^{+} \mathrm{e}^{-}$mode. We extract the cross section under the assumption that the theory describes correctly the shape of the kinematical distributions of the dominant background from Drell-Yan production in association with two jets 
The signal-to-background ratio for the cross section measurement is small. In order to confirm the presence of a signal, two methods of signal extraction are employed and two different jet algorithms are used. While providing a similar performance, these two types of jet algorithms use different methods to combine the information from the subdetectors, different energy corrections, and different methods to account for the energy from the additional minimum-bias events (pileup).

In a separate study, measurements of the hadronic activity in Drell-Yan events are presented. These include the level of hadronic activity in the rapidity interval between the two tagging jets and the properties of multi-jets in events with a $\mathrm{Z}$ boson.

The plan of the paper is as follows: in section 2 we describe the CMS detector, reconstruction, and event simulation; in section 3 we discuss the event selections; sections 4 and 5 are devoted to the study of the hadronic activity in Drell-Yan events; in section 6 we present the measurement of the cross section for the EW Zjj production; finally, in section 7 we summarize our main results.

\section{CMS detector, reconstruction, and event simulation}

A detailed description of the CMS detector can be found in ref. [16]. The CMS experiment uses a right-handed coordinate system, with the origin at the nominal interaction point, the $x$ axis pointing to the center of the LHC ring, the $y$ axis pointing up, and the $z$ axis along the counterclockwise-beam direction as viewed from above. The polar angle $\theta$ is measured from the positive $z$ axis and the azimuthal angle $\phi$ is measured in the $x-y$ plane. The pseudorapidity $\eta$ is defined as $-\ln [\tan (\theta / 2)]$, which equals the rapidity $y=\ln \left[\left(E+p_{z}\right) /\left(E-p_{z}\right)\right]$ for massless particles.

The central feature of the CMS apparatus is a superconducting solenoid of $6 \mathrm{~m}$ internal diameter providing a field of $3.8 \mathrm{~T}$. Within the field volume are a silicon pixel and strip tracker, a crystal electromagnetic calorimeter (ECAL), and a brass/scintillator hadron calorimeter (HCAL) providing coverage for pseudorapidities $|\eta|<3$. The forward calorimeter modules extend the coverage of hadronic jets up to $|\eta|<5$. Muons are measured in gas-ionization detectors embedded in the steel magnetic flux return yoke.

The first level (L1) of the CMS trigger system, composed of custom hardware processors, uses information from the calorimeters and the muon detectors to select the most interesting events. The high-level-trigger processor farm further decreases the event rate from $\sim 100 \mathrm{kHz}$ of $\mathrm{L} 1$ accepts to a few hundred $\mathrm{Hz}$, before data storage.

Muons are reconstructed [17] by fitting trajectories based on hits in the silicon tracker and the muon system. Electrons are reconstructed [18] from clusters of energy deposits in the ECAL matched to tracks in the silicon tracker.

Two different types of jets are used in the analysis: jet-plus-track (JPT) and particleflow (PF) jets [19]. The JPT jets are reconstructed calorimeter jets whose energy response and resolution are improved by incorporating tracking information according to the JPT algorithm [20]. Calorimeter jets are first reconstructed from energy deposits in the calorimeter towers clustered with the anti- $k_{\mathrm{T}}$ jet algorithm $[21,22]$ with a distance parameter of 0.5. Charged-particle tracks are associated with each jet, based on the spatial separation 
in $\eta$ - $\phi$ between the jet axis and the track momentum vector measured at the interaction vertex. The associated tracks are projected onto the surface of the calorimeter and classified as in-cone tracks if they point within the jet cone around the jet axis. The tracks bent out of the jet cone due to the magnetic field are classified as out-of-cone tracks. The momenta of the charged tracks are used to improve the measurement of the energy of the associated calorimeter jet. For in-cone tracks the expected average energy deposition in the calorimeters is subtracted and the energy of the tracks (assuming that they are charged pions) is added to the jet energy. For out-of-cone tracks the energy of the tracks is added directly to the jet energy. The direction of the jet is re-calculated with the tracks. As a result of the JPT algorithm, both the energy and the direction of the jet are improved.

The CMS particle flow algorithm [23, 24] combines the information from all relevant CMS sub-detectors to identify and reconstruct particle candidates in the event: muons, electrons, photons, charged hadrons, and neutral hadrons. Charged hadrons are reconstructed from tracks in the tracker. Photons and neutral hadrons are reconstructed from energy clusters in the ECAL and HCAL, respectively, that are separate from the extrapolated position of tracks. A neutral particle overlapping with charged particles in the calorimeters is identified from a calorimeter energy excess with respect to the sum of the associated track momenta. Particle flow jets (PF jets) are reconstructed using the anti- $k_{\mathrm{T}}$ jet algorithm with a distance parameter of 0.5 , clustering particles identified by the particle flow algorithm.

The signal process for this analysis is the electroweak production of a dilepton pair in association with two jets (EW $\ell \ell \mathrm{jj}, \ell=\mathrm{e}, \mu)$. It is simulated with MADGRAPH version 5 [25, 26] interfaced with PYTHIA 6.4.25 [27] for parton showering (PS) and hadronization. The CTEQ6L1 parton distribution functions [28] are used in the event generation by MadGraph. The electroweak pp $\rightarrow \ell \ell j \mathrm{j}$ processes in MAdGraPh include WZ production where the $\mathrm{W}$ boson decays into two quarks and $\mathrm{ZZ}$ production where one of the $\mathrm{Z}$ bosons decays into two quarks. The requirement $m_{\mathrm{jj}}>120 \mathrm{GeV}$ applied at the MADGRAPH generation level reduces the contribution from these processes to a negligible level in the defined signal phase space. For the leading order generators, $\mathrm{j}$ stands for partons. For next-to-leading order calculations, a jet algorithm is applied to the final state partons and $\mathrm{j}$ stands for the parton jets.

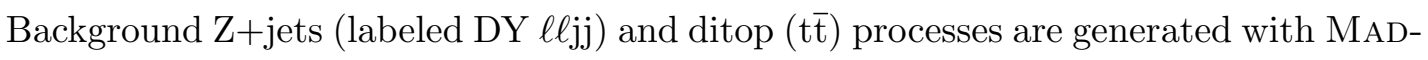
GRAPH via a matrix element (ME) calculation that includes up to four jets at parton level. The ME and parton shower (ME-PS) matching is performed following the ktMLM prescription [26]. The generation of the DY $\ell \ell j \mathrm{j}$ background does not include the electroweak production of the $\mathrm{Z}$ boson with two jets. The diboson production processes WW, WZ, and ZZ are generated with PYTHIA.

The MCFM program [29] is also used for the evaluation of the theoretical uncertainty of the DY $\ell \ell \mathrm{jj}$ background predictions. The dynamic scale $\mu_{0}=\sum_{i=1}^{n} p_{\mathrm{T}}^{i}$ with $n$ final state particles (partons, not jets; $n=4,5$ ) is used with the QCD factorization and renormalization scales set equal, $\mu_{\mathrm{F}}=\mu_{\mathrm{R}}=\mu_{0}$.

Generated events are processed through the full CMS detector simulation based on GEAnt4 [30, 31], followed by a detailed trigger emulation, and the standard event recon- 
struction. Minimum-bias events are superimposed upon the hard interaction to simulate the effects of additional interactions per beam crossing (pileup). The multiplicity distribution of the pileup events in the simulation is matched with that observed in data. The PYTHIA parameters for the underlying event were set according to the $\mathrm{Z} 2$ tune [32].

The signal cross section per lepton flavor, at next-to-leading order (NLO), is calculated to be $\sigma_{\mathrm{NLO}}(\mathrm{EW} \ell \ell \mathrm{jj})=166 \mathrm{fb}$. The calculation is carried out with the VBFNLO program [33] with the factorization and renormalization scales set to $\mu_{\mathrm{R}}=\mu_{\mathrm{F}}=90 \mathrm{GeV}$ and with CT10 parton distribution functions [34]. The calculation is performed in the following kinematical region: a dilepton invariant mass, $m_{\ell \ell}$ above $50 \mathrm{GeV}$, jet transverse momentum $p_{\mathrm{T}}^{\mathrm{j}}>25 \mathrm{GeV}$, jet pseudorapidity $\left|\eta^{\mathrm{j}}\right|<4$, and dijet invariant mass $m_{\mathrm{jj}}>120 \mathrm{GeV}$. The kinematic distributions for the signal generated by VBFNLO at leading order agree with those produced by the MADGRAPH generator.

The interference effects between EW and DY $\ell$ jj production processes are evaluated with the MADGraph, SHERPA [35], COMPHEP [36], and vBFnlo programs by the authors of these programs and were found to be negligible.

\section{Event selection}

For the muon channel, the candidate events were selected by a trigger that required the presence of two muons. The requirement applied by the trigger on the muon transverse momenta changed with increasing instantaneous luminosity. As a consequence, the analyzed data sample is divided into three sets corresponding to the following different thresholds: (i) both muons have $p_{\mathrm{T}}^{\mu}>7 \mathrm{GeV}$, (ii) $p_{\mathrm{T}}^{\mu_{1}}>13 \mathrm{GeV}$ and $p_{\mathrm{T}}^{\mu_{2}}>8 \mathrm{GeV}$, and (iii) $p_{\mathrm{T}}^{\mu_{1}}>17 \mathrm{GeV}$ and $p_{\mathrm{T}}^{\mu_{2}}>8 \mathrm{GeV}$. Events in the electron channel were selected by a trigger that required the presence of two electrons with $p_{\mathrm{T}}^{\mathrm{e}_{1}}>17 \mathrm{GeV}$ and $p_{\mathrm{T}}^{\mathrm{e}_{2}}>8 \mathrm{GeV}$.

Offline, the muon candidates used in the analysis are identified by an algorithm [17], which starts from the tracks measured in the muon chambers, and then matches and combines them with the tracks reconstructed in the inner tracker. Muons from the in-flight decays of hadrons and punch-through particles are suppressed by applying a requirement on the goodness-of-fit over the number of degrees of freedom, $\chi^{2} /$ dof $<10$, of the global fit including the hits in the tracker and muon detectors.

In order to ensure a precise estimate of momentum and impact parameter, only tracks with more than 10 hits in the inner tracker and at least one hit in the pixel detector are used. We require hits in at least two muon detectors, to ensure a precise momentum estimate at the trigger level, and to suppress remaining background from misidentified muon candidates. Cosmic muons are rejected by requiring a transverse impact parameter distance to the beam spot position of less than $2 \mathrm{~mm}$. These selection criteria provide an efficiency of $96 \%$ for prompt muons with $p_{\mathrm{T}}>20 \mathrm{GeV}$. The efficiency is defined as a ratio where the denominator is the number of generated muons with $p_{\mathrm{T}}>20 \mathrm{GeV}$ within the geometrical acceptance and the numerator is the number of those muons that pass the selection criteria described above.

The electron candidates are required to pass a set of criteria which is $90 \%$ efficient for prompt electrons with $p_{\mathrm{T}}>20 \mathrm{GeV}$ [18]. The electron identification variables used 
in the selection are (i) the spatial distance between the track and the associated ECAL cluster, (ii) the size and the shape of the shower in ECAL, and (iii) the hadronic leakage. The track transverse impact parameter is used to discriminate electrons from conversions. Tracks from conversions have, on average, a greater distance to the beam axis. In order to reject electrons from conversions, candidates are allowed to have at most one missing hit among those expected in the innermost tracker layers.

Electrons and muons from heavy-flavor decays and contained in hadronic jets are suppressed by imposing a restriction on the presence of additional tracks around their momentum direction. The additional tracks are summed in a cone of radius $\Delta R=$ $\sqrt{(\Delta \eta)^{2}+(\Delta \phi)^{2}}<0.3$ around the lepton candidate. Only tracks consistent with originating from the vertex corresponding to the hardest proton-proton scattering are used in the evaluation, so as to be insensitive to contributions from pileup interactions in the same bunch crossing. A relative isolation variable, $I_{\mathrm{trk}}=\sum p_{\mathrm{T}}^{\mathrm{trk}} / p_{\mathrm{T}}^{\ell}$, is evaluated for each lepton.

The dimuon channel selection " $\mathrm{Z}_{\mu \mu}$ " is defined by the following set of requirements: the two highest- $p_{\mathrm{T}}$ muons must have $p_{\mathrm{T}}>20 \mathrm{GeV},|\eta|<2.4$, and must satisfy the muon quality criteria described above. The muons are required to have opposite charge and have a relative isolation of $I_{\text {trk }}<0.1$. The dimuon invariant mass is required to be within $\pm 15 \mathrm{GeV}$ of the $\mathrm{Z}$ boson mass $m_{\mathrm{Z}}=91.2 \mathrm{GeV}$.

The following set of requirements define the " $\mathrm{Z}_{\mathrm{ee}}$ " dielectron selection: the two higest$p_{\mathrm{T}}$ electrons must have $p_{\mathrm{T}}>20 \mathrm{GeV},|\eta|<2.4$, and satisfy the electron quality criteria described previously. The electrons are required to have opposite charge and relative isolation criteria of $I_{\mathrm{trk}}<0.1$.

The dielectron invariant mass is required to be within $\pm 20 \mathrm{GeV}$ of $m_{\mathrm{Z}}$, a larger mass range than that for $m_{\mu \mu}$ since the dielectron Z-peak is wider because of electron bremsstrahlung effects in the tracker material.

The two highest- $p_{\mathrm{T}}$ leading jets in the event with $\left|\eta_{j}\right|<4.7$ (labeled $\mathrm{j}_{1}$ and $\mathrm{j}_{2}$ ) are selected as the tagging jets. The selection criteria are optimized by maximizing the signal significance defined as $N_{S} / \sqrt{N_{B}}$, where $N_{S}$ and $N_{B}$ are the number of signal and background events passing the selection criteria, expected from the Monte Carlo (MC) signal and DY samples, with an integrated luminosity of $5 \mathrm{fb}^{-1}$. The requirements on the momentum and pseudorapidity of the tagging jets $\left(p_{\mathrm{T}}^{\mathrm{j}_{1}}, p_{\mathrm{T}}^{\mathrm{j}_{2}}, \eta_{\mathrm{j}}\right)$, the dijet invariant mass $\left(m_{\mathrm{j}_{1} \mathrm{j}_{2}}\right)$, and the $\mathrm{Z}$ boson rapidity in the rest frame of the tagging jets $y^{*}=y_{Z}-0.5\left(y_{\mathrm{j}_{1}}+y_{\mathrm{j}_{2}}\right)$ are varied in order to reach maximum signal significance. The optimized selection criteria shown in table 1, with the corresponding selection labels, result in an expected signal significance of about three for an integrated luminosity of $5 \mathrm{fb}^{-1}$, for each of the dilepton channels.

The signal efficiencies for the $Z_{\mu \mu}$ selection without additional requirements, with the tagging jet requirement $\mathrm{TJ} 1$, with the $\mathrm{TJ} 1$ and the $\mathrm{Z}$ boson rapidity requirement $\mathrm{YZ}$, and with the TJ1, YZ, and TJ2 requirements are $0.36,0.23,0.17$, and 0.06 , respectively. These efficiencies are valid both in the case of JPT and PF jet reconstruction. The signal efficiencies for the dielectron channel are respectively $0.33,0.21,0.16$, and 0.06 . The efficiency is defined as a ratio where the denominator is the number of signal events generated by MADGRAPH with $m_{\mathrm{jj}}>120 \mathrm{GeV}$ and the numerator is the number of events that passed the selections described above. 


\begin{tabular}{|c|c|}
\hline \multicolumn{2}{|c|}{ Tagging jet selections } \\
\hline TJ1 & $p_{\mathrm{T}}^{\mathrm{j}_{1}}>65 \mathrm{GeV}, p_{\mathrm{T}}^{\mathrm{j}_{2}}>40 \mathrm{GeV},\left|\eta_{\mathrm{j}}\right|<3.6$ \\
TJ2 & $m_{\mathrm{j}_{1} \mathrm{j}_{2}}>600 \mathrm{GeV}$ \\
\hline \multicolumn{2}{|c|}{ Z boson rapidity selection } \\
\hline $\mathrm{YZ}$ & $\left|y^{*}\right|<1.2$ \\
\hline
\end{tabular}

Table 1. The optimized selection criteria with the corresponding selection labels.
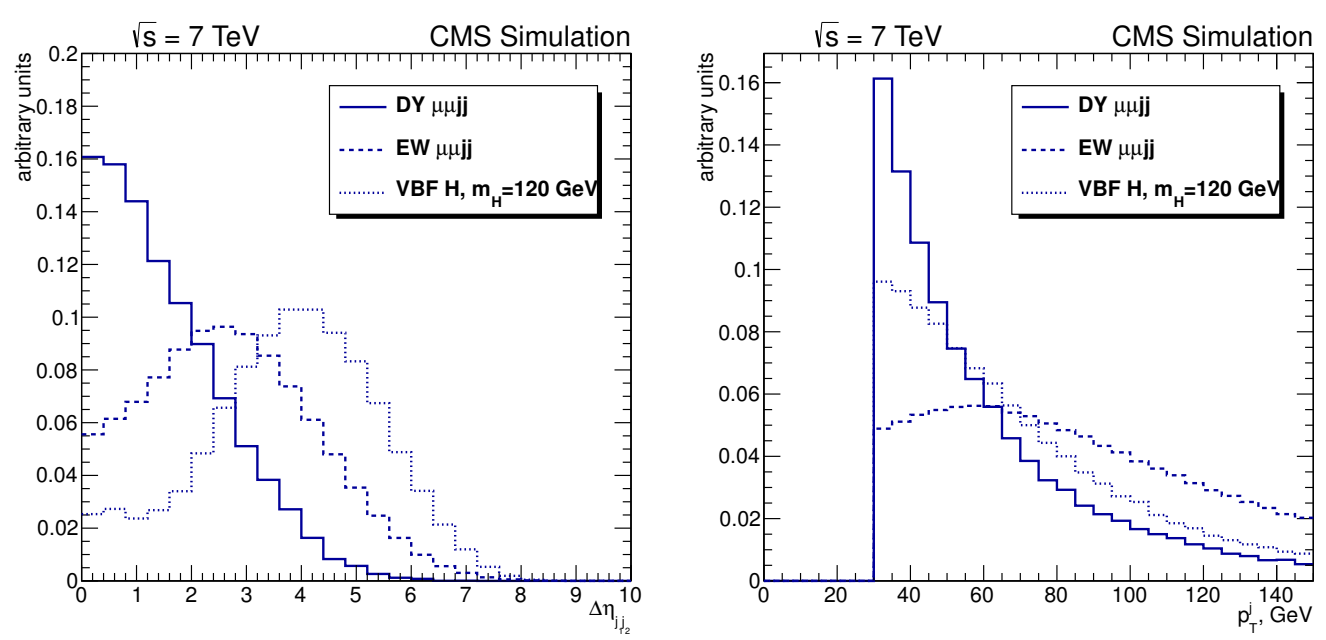

Figure 3. Distribution of the absolute difference in the pseudorapidity of the tagging jets, $\Delta \eta_{\mathrm{j}_{1} \mathrm{j}_{2}}=$ $\left|\eta_{\mathrm{j}_{1}}-\eta_{\mathrm{j}_{2}}\right|$ (left) and the tagging jet $p_{\mathrm{T}}$ for both jets, $\mathrm{j}_{1}$ and $\mathrm{j}_{2}$ (right) for the DY $\mu \mu \mathrm{jj}$, EW $\mu \mu \mathrm{jj}$, and VBF Higgs boson production processes.

The above event selection criteria are different from those suggested for Higgs boson searches in the VBF channel [5-8]. In particular, higher $p_{\mathrm{T}}$ thresholds are used on the tagging jets, the rapidity separation between the tagging jets is not used, and a central jet veto is not applied. This is because the kinematics for EW Zjj production and VBF Higgs boson production are different. The former includes two additional contributions, bremsstrahlung and multiperipheral processes, as shown in figure 1. These additional processes and the interference between them lead to higher average jet transverse momenta in comparison to the VBF production process alone. This is due to the fact that the EW $\ell$ jj process involves transversely polarized $\mathrm{W}$ bosons, while the main contribution to VBF boson production involves longitudinally polarised $\mathrm{W}$ bosons. Figure 3 shows the simulated distributions of the absolute pseudorapidity difference of the two tagging jets, $\Delta \eta_{\mathrm{j}_{1} \mathrm{j}_{2}}=\left|\eta_{\mathrm{j}_{1}}-\eta_{\mathrm{j}_{2}}\right|$ (left), and the tagging jets $p_{\mathrm{T}}$ (right) for the DY $\mu \mu \mathrm{jj}$, the EW $\mu \mu \mathrm{jj}$, and the VBF Higgs boson production processes. The $m_{\mathrm{j}_{1} \mathrm{j}_{2}}$ distributions for EW $\mu \mu \mathrm{jj}$ production and VBF Higgs boson production processes are very similar and are not shown here.

The event selection is performed with JPT and PF jets in the dimuon channel and with $\mathrm{PF}$ jets in dielectron channel. Table 2 shows the event yield after each selection step in the 


\begin{tabular}{|c|c|c|c|c|c|c|c|c|}
\hline Selection & Jet type & Data & $E W \ell \ell j \mathrm{j}$ & DY $\ell \ell j \mathrm{j}$ & $\mathrm{t} \overline{\mathrm{t}}$ & WW & $\mathrm{WZ}$ & $\mathrm{ZZ}$ \\
\hline $\mathrm{Z}_{\mu \mu}$ & & $1.7 \times 10^{6}$ & 460 & $1.7 \times 10^{6}$ & 1400 & 300 & 1300 & 850 \\
\hline \multirow[t]{2}{*}{ requirement TJ1 } & JPT & 25000 & 290 & 26000 & 690 & 5.2 & 180 & 120 \\
\hline & $\mathrm{PF}$ & 26000 & 280 & 26000 & 680 & 5.3 & 170 & 110 \\
\hline \multirow[t]{2}{*}{ requirement YZ } & JPT & 15000 & 210 & 16000 & 590 & 3.4 & 98 & 83 \\
\hline & $\mathrm{PF}$ & 16000 & 200 & 16000 & 580 & 3.4 & 93 & 76 \\
\hline \multirow[t]{2}{*}{ requirement TJ2 } & JPT & 600 & 74 & 600 & 14 & 0 & 2.2 & 1.3 \\
\hline & $\mathrm{PF}$ & 640 & 72 & 610 & 14 & 0 & 2.4 & 1.2 \\
\hline
\end{tabular}

Table 2. Event yields in the $\mu^{+} \mu^{-}$channel after each selection step for the data, the signal Monte Carlo and the backgrounds. The expected contributions from the signal and background processes are evaluated from simulation, for $5 \mathrm{fb}^{-1}$ of integrated luminosity.

\begin{tabular}{|c|c|c|c|c|c|c|c|}
\hline selection & data & EW $\ell \ell \mathrm{jj}$ & $\mathrm{DY} \ell \ell \mathrm{jj}$ & $\mathrm{t} \overline{\mathrm{t}}$ & $\mathrm{WW}$ & $\mathrm{WZ}$ & $\mathrm{ZZ}$ \\
\hline $\mathrm{Z}_{\mathrm{ee}}$ & $1.5 \times 10^{6}$ & 410 & $1.5 \times 10^{6}$ & 1600 & 340 & 1100 & 720 \\
requirement TJ1 & 24000 & 270 & 23000 & 880 & 6.0 & 150 & 97 \\
requirement YZ & 15000 & 200 & 15000 & 760 & 3.7 & 90 & 68 \\
requirement TJ2 & 560 & 67 & 550 & 17 & 0.3 & 2.5 & 1.0 \\
\hline
\end{tabular}

Table 3. Event yields in the $\mathrm{e}^{+} \mathrm{e}^{-}$channel after each selection step for the data, the signal Monte Carlo and the backgrounds. The expected contributions from the signal and background processes are evaluated from simulation, for $5 \mathrm{fb}^{-1}$ of integrated luminosity.

$\mu^{+} \mu^{-}$channel. The observed and expected number of events from signal and background processes are shown for the different selection requirements. The two jet algorithms result in similar yields. Table 3 shows the event yields after each selection step with PF jets in the $\mathrm{e}^{+} \mathrm{e}^{-}$channel.

The uncertainty on the estimation of the dominant DY $\ell \ell \mathrm{jj}$ background from simulation is comparable with the expected number of signal events. The signal can therefore only be extracted by analyzing the distributions that are most sensitive to the difference between the signal and backgrounds.

Distributions for data and simulation after the $\mathrm{Z}_{\mu \mu}$ selection and jet tagging requirement TJ1 are shown in figures 4 and 5 . In these and the following figures the histograms

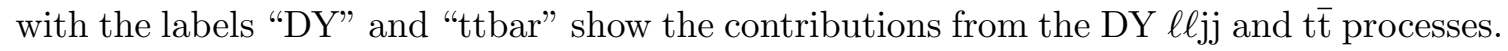
The labels "WZ", "ZZ", and "WW" apply to the diboson production processes WZ, ZZ, and WW. The label "EW" shows the contribution from the signal process, EW Zjj.

The $p_{\mathrm{T}}^{\mathrm{j}_{1}}$ and $p_{\mathrm{T}}^{\mathrm{j}_{2}}$ distributions obtained with JPT jets are shown in figure 4 . The absolute difference in the pseudorapidity of the two tagging JPT jets, and the dimuon $p_{\mathrm{T}}$ are shown in figure 5. The expected contributions from the signal and background processes are evaluated from simulation. The bottom panel in the figures shows the ratio of the data to the expected contribution of the signal plus background together with the 

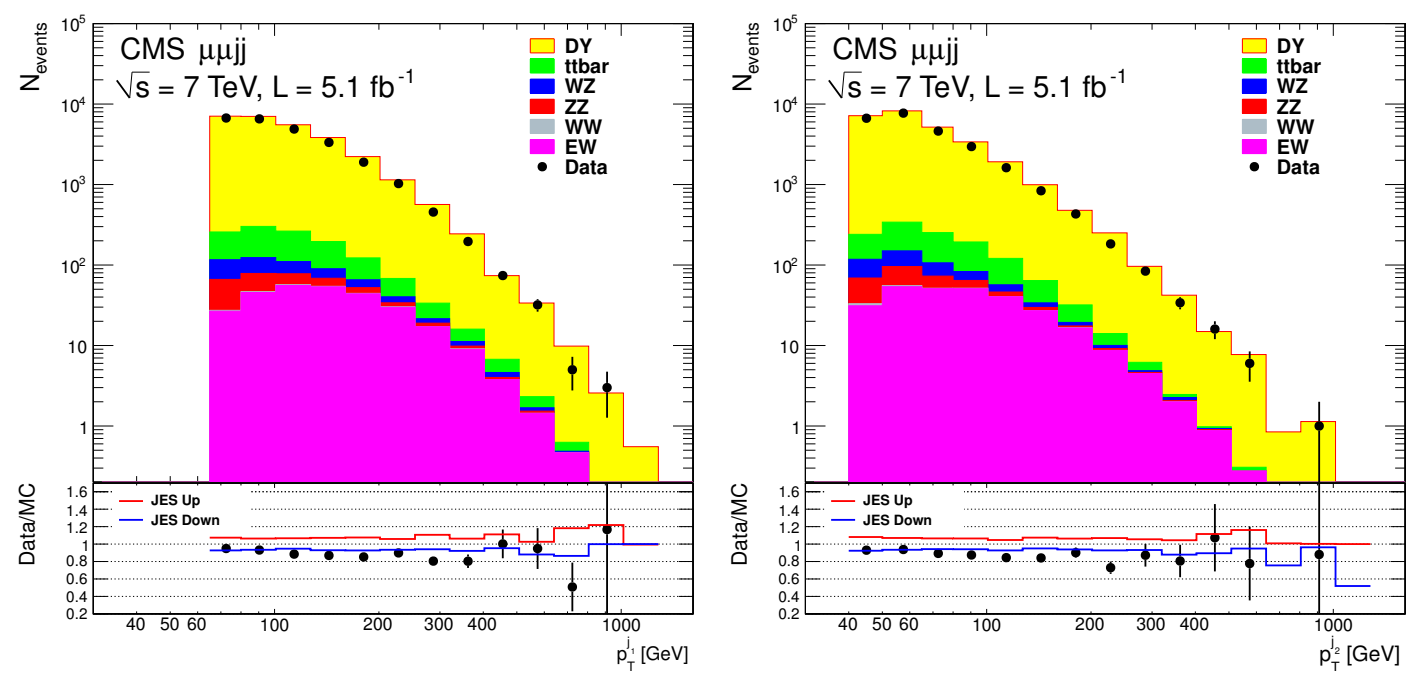

Figure 4. The $p_{\mathrm{T}}^{\mathrm{j}_{1}}$ (left) and $p_{\mathrm{T}}^{\mathrm{j}_{2}}$ (right) distributions after applying the $\mathrm{Z}_{\mu \mu}$ selection and the jet tagging requirement TJ1. The expected contributions from the signal and background processes are evaluated from simulation. The bottom panels show the ratio of data over the expected contribution of the signal plus background. The region between the two lines with the labels JES Up and JES Down shows the $1 \sigma$ uncertainty of the simulation prediction due to the jet energy scale uncertainty. The data points are shown with the statistical uncertainties.

statistical uncertainties. The region between the two lines with the labels JES Up and JES Down shows the $\pm 1 \sigma$ uncertainty of the simulation prediction due to the jet energy scale (JES) uncertainty.

The ratio of the data to the expected contribution of the signal plus background is systematically below unity and outside the $1 \sigma$ JES uncertainty in some regions. However, it is consistent with unity within the systematic uncertainty in the MADGRAPH simulation of the dominant DY $\ell \ell \mathrm{jj}$ background. The systematic uncertainty due to the QCD scale is expected to be between the uncertainty given by the NLO and LO calculations, which are $8 \%$ and $25 \%$, respectively, as calculated by the MCFM program. The choice of the QCD scale is discussed in section 6.2.

Figures 4 and 5 illustrate the overall level of agreement between data and simulation. It is evident from the figures that the signal fraction is small; this is why the extraction of the signal requires the special methods described in section 6 .

In sections 4 and 5 we describe the measurements of the hadronic activity in the rapidity interval between the tagging jets and the measurements of the radiation patterns in multijet events in association with a $\mathrm{Z}$ boson. The selected data sample is dominated by DY $\ell \ell j j$ events which are referred to as "DY Zjj events" in the following two sections.

\section{Hadronic activity in the rapidity interval between tagging jets}

A veto on the hadronic activity in the rapidity interval between the VBF tagging jets has been proposed [5-8] as a tool to suppress backgrounds in the searches for a Higgs boson produced in VBF. In the following, a study of the hadronic activity in this rapidity interval 

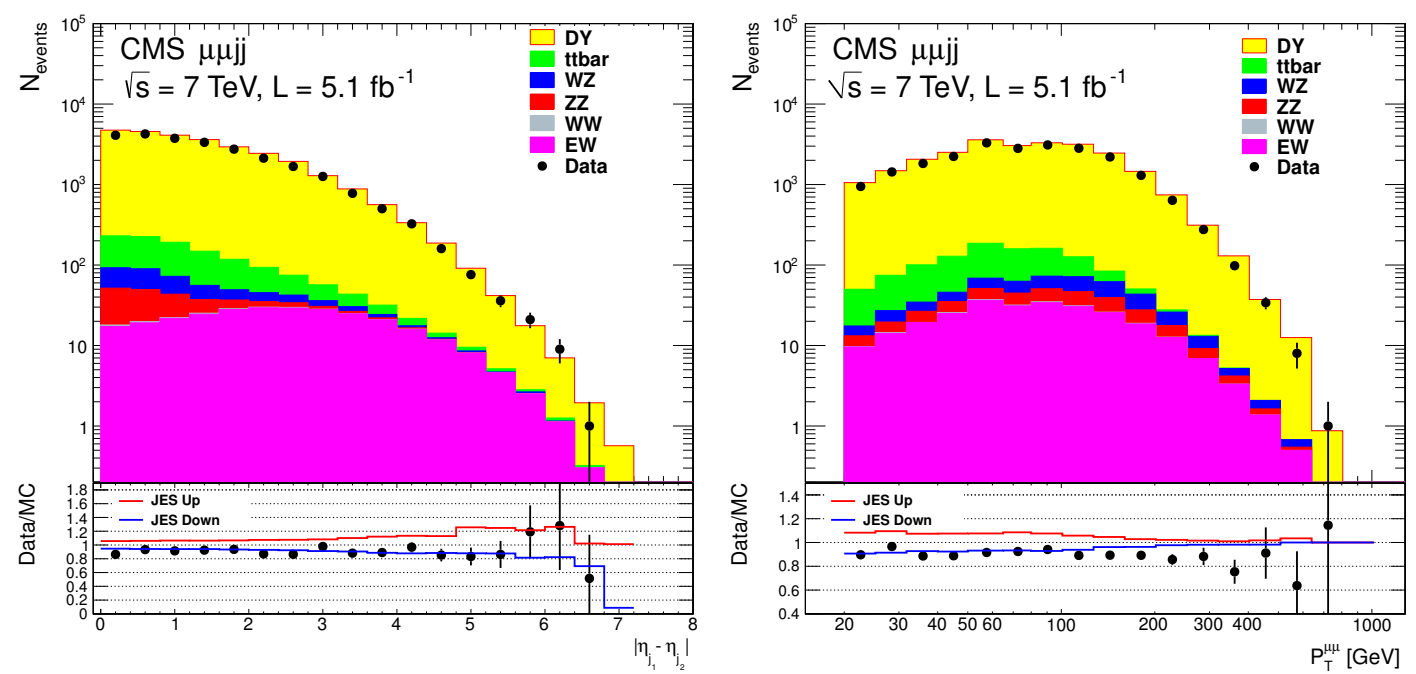

Figure 5. The absolute difference in the pseudorapidity of the two tagging jets (left), and the dimuon $p_{\mathrm{T}}$ (right) after the $\mathrm{Z}_{\mu \mu}$ selection and the tagging jet requirement TJ1. The expected contributions from the signal and background processes are evaluated from simulation. The bottom panels show the ratio of data over the expected contribution of the signal plus background. The region between the two lines with the labels JES Up and JES Down shows the $1 \sigma$ uncertainty of the simulation prediction due to the jet energy scale uncertainty. The data points are shown with the statistical uncertainties.

is presented. Although a veto is not used on the hadronic activity to select the EW $\ell$ ljj process, the studies provided in this section and in section 5 can be considered as a test of the agreement between the data and the simulation for the dominant DY $\ell \ell j j$ background. The data sample is selected with the $Z_{\mu \mu}$ and $Z_{\text {ee }}$ requirements described in section 3 . The requirements on the jets are described in this section and in section 5 .

\subsection{Central hadronic activity measurement using jets}

The hadronic activity in the rapidity interval between the tagging jets is studied as a function of the pseudorapidity separation between the tagging jets, the $p_{\mathrm{T}}$ threshold of the tagging jets, and the dijet invariant mass, $m_{\mathrm{j}_{1} j_{2}}$. The hadronic activity is measured through the efficiency of the central jet veto, defined as the fraction of selected events with no third jet $\left(\mathrm{j}_{3}\right)$ with $p_{\mathrm{T}}^{\mathrm{j}_{3}}>20 \mathrm{GeV}$ in the pseudorapidity interval between the tagging jets:

$$
\eta_{\min }^{\mathrm{tag} j e t}+0.5<\eta_{\mathrm{j} 3}<\eta_{\max }^{\mathrm{tag} j e t}-0.5 \quad \text { and } \quad\left|\eta_{\mathrm{j}_{3}}\right|<2.0
$$

where $\eta_{\min }^{\text {tag jet }}\left(\eta_{\max }^{\mathrm{tag} \text { jet }}\right)$ is the minimal (maximal) pseudorapidity of the tagging jet. The central jets from pileup interactions are suppressed with the tracker information.

Tables 4 and 5 show the efficiencies measured from the data and those obtained from the MADGRAPH DY $\ell$ jj simulation for the different requirements on the $p_{\mathrm{T}}$ of the tagging jets, the pseudorapidity separation between them, and their invariant mass. The measured

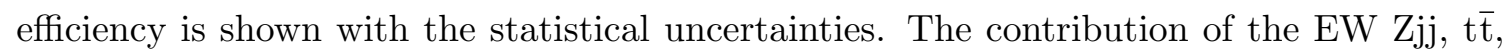
and diboson processes is not subtracted from the data measurements since it does not 


\begin{tabular}{|c|c|c|c|}
\hline$p_{\mathrm{T}}^{\mathrm{j}_{1}\left(\mathrm{j}_{2}\right)}$ & $>25 \mathrm{GeV}$ & $>35 \mathrm{GeV}$ & $>45 \mathrm{GeV}$ \\
\hline data & $0.78 \pm 0.01$ & $0.68 \pm 0.01$ & $0.63 \pm 0.02$ \\
simulation & 0.80 & 0.71 & 0.66 \\
\hline
\end{tabular}

Table 4. Efficiency of the central jet veto with $p_{\mathrm{T}}^{\mathrm{j}_{3}}>20 \mathrm{GeV}$ for three different selections on the tagging jets for a pseudorapidity separation of $\Delta \eta_{\mathrm{j}_{1} \mathrm{j}_{2}}>3.5$ measured in data and predicted by the MADGRAPH simulation. The quoted uncertainty is statistical only.

\begin{tabular}{|c|c|c|c|}
\hline$\Delta \eta_{\mathrm{j}_{1} \mathrm{j}_{2}}$ & $>2.5$ & $>3.5$ & $>4.5$ \\
\hline $\begin{array}{c}\text { data } \\
\text { simulation }\end{array}$ & $0.71 \pm 0.01$ & $0.68 \pm 0.01$ & $0.66 \pm 0.02$ \\
\hline \multicolumn{4}{|c|}{ with $m_{\mathrm{j}_{1} \mathrm{j}_{2}}>700 \mathrm{GeV}$ selection } \\
\hline data & $0.56 \pm 0.03$ & $0.58 \pm 0.03$ & $0.62 \pm 0.04$ \\
simulation & 0.56 & 0.57 & 0.58 \\
\hline
\end{tabular}

Table 5. Efficiency of the central jet veto with $p_{\mathrm{T}}^{\mathrm{j}_{3}}>20 \mathrm{GeV}$ and $p_{\mathrm{T}}^{\mathrm{j}_{1}\left(\mathrm{j}_{2}\right)}>30 \mathrm{GeV}$ for three different selections for $\Delta \eta_{\mathrm{j}_{1} \mathrm{j}_{2}}$ with and without the selection on $m_{\mathrm{j}_{1} \mathrm{j}_{2}}$, measured in data and predicted by the MAdGraph simulation. The quoted uncertainty on the data efficiency is only statistical.

change the measured efficiency within the uncertainties. The efficiencies shown in table 5 are evaluated for the pseudorapidity interval

$$
\eta_{\min }^{\mathrm{tag} j e t}<\eta_{\mathrm{j}_{3}}<\eta_{\max }^{\mathrm{tag} \text { jet }} \quad \text { and } \quad\left|\eta_{\mathrm{j}_{3}}\right|<2.0 .
$$

The veto efficiencies obtained from data and the MADGRAPH simulation are in good agreement.

\subsection{Central hadronic activity measurement with track jets}

As the hadronic activity in the rapidity interval between the tagging jets is expected to be small (soft) in the case of a purely electroweak Zjj production, the contribution from any additional pileup interaction in the event needs to be avoided or carefully subtracted. For this reason, an additional study of the interjet hadronic activity is performed using only charged tracks that clearly originate from the hard-scattering vertex in the event.

For this study a collection of tracks is built with reconstructed high-purity tracks [37] with $p_{\mathrm{T}}>300 \mathrm{MeV}$ that are uniquely associated with the main primary vertex in the event. Tracks associated with the two leptons or with the tagging jets are not included. The association between the tracks and the reconstructed primary vertices is carried out by minimizing the longitudinal distance $d_{z}(\mathrm{PV})$ between the primary vertex $(\mathrm{PV})$ and the point of closest approach of the track helix to that PV. The association is required to satisfy $d_{z}(\mathrm{PV})<2 \mathrm{~mm}$ and $d_{z}(\mathrm{PV})<3 \delta d_{z}(\mathrm{PV})$, where $\delta d_{z}(\mathrm{PV})$ is the uncertainty on $d_{z}(\mathrm{PV})$. The main primary vertex in the event is chosen to be that with the largest scalar sum of transverse momenta, for all tracks used to reconstruct it. 

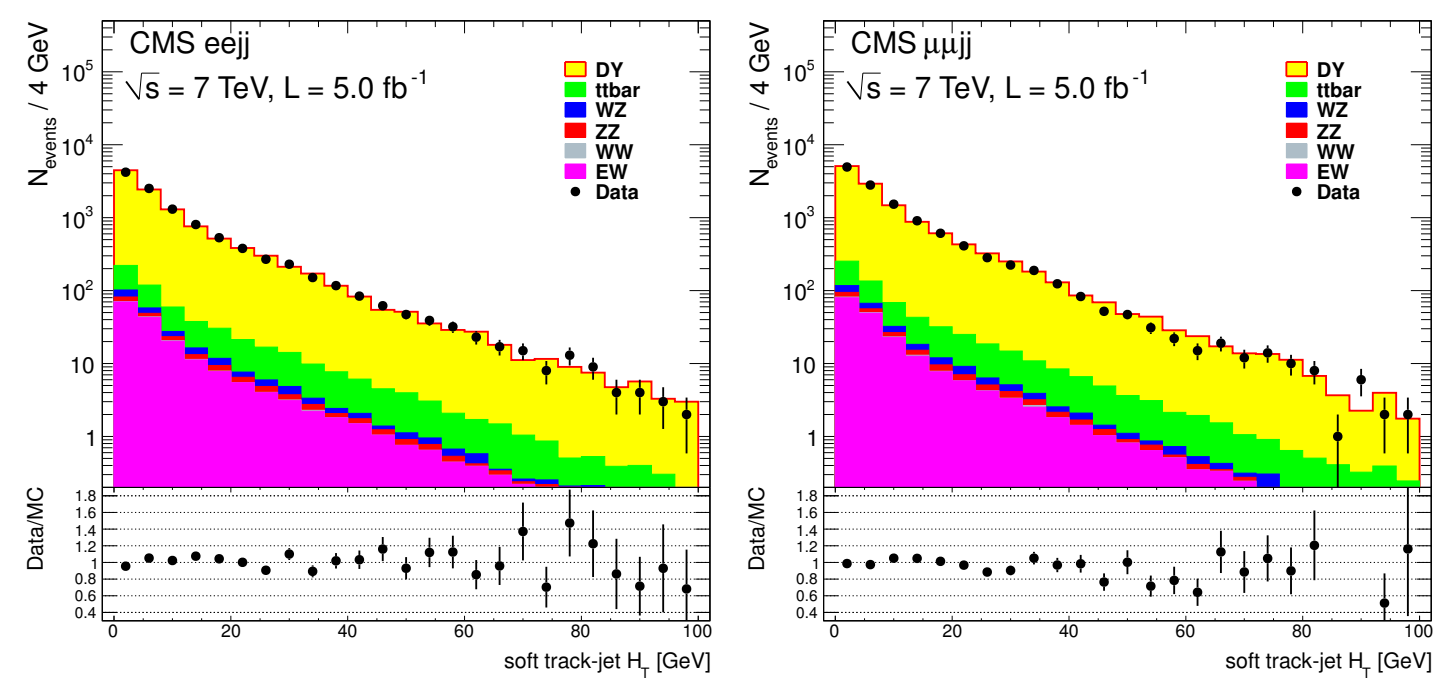

Figure 6. The $H_{\mathrm{T}}$ distribution of the three leading soft track jets in the pseudorapidity interval between the tagging jets with $p_{\mathrm{T}}^{\mathrm{j}_{1}, \mathrm{j}_{2}}>65,40 \mathrm{GeV}$ in DY Zjj events for dielectron (left) and dimuon (right) channels. The bottom panels show the corresponding data/MC ratios. The data points are shown with the statistical uncertainties.

A collection of "soft track jets" is built by clustering the tracks with the anti- $k_{\mathrm{T}}$ clustering algorithm [22] with a distance parameter of 0.5. The use of track jets represents a clean and well understood method [38] to reconstruct jets with energy as low as a few $\mathrm{GeV}$. Crucially, these jets are not affected by pileup because of the association of their tracks with the hard-scattering vertex [39].

For the purpose of studying the central hadronic activity between the tagging jets, only soft track jets with pseudorapidity $\eta_{\min }^{\text {tag jet }}+0.5<\eta<\eta_{\max }^{\text {tag jet }}-0.5$ are considered. The scalar sum $\left(H_{\mathrm{T}}\right)$ of the transverse momenta of up to three soft track jets is used as a monitor of the hadronic activity in the rapidity interval between the two jets. The soft $H_{\mathrm{T}}$ distribution is shown in figure 6 for DY Zjj events for $p_{\mathrm{T}}^{\mathrm{j}_{1} \mathrm{j}_{2}}>65,40 \mathrm{GeV}$. The expectations from the simulation for the hadronic activity between the tagging jets are in good agreement with the data.

The evolution of the average $H_{\mathrm{T}}$ for $\mathrm{DY} \mathrm{Zjj}$ jets events as a function of the dijet invariant mass $m_{\mathrm{j}_{1} \mathrm{j}_{2}}$ and the pseudorapidity difference $\Delta \eta_{\mathrm{j}_{1} \mathrm{j}_{2}}$ between the tagging jets is shown in figure 7 . For better visibility the symbols at each measured point are slightly displaced along the $\mathrm{x}$ axis. Good agreement is observed between the simulation and the data for the different mass and pseudorapidity intervals.

\section{Measurements of the radiation patterns in multijet events in associa- tion with a $\mathrm{Z}$ boson}

In hard multijet events in association with a $\mathrm{Z}$ boson, the observables referred to as "radiation patterns" are:

- the number of jets $N_{\mathrm{j}}$;

- the total scalar sum $\left(H_{\mathrm{T}}\right)$ of jets with $|\eta|<4.7$; 

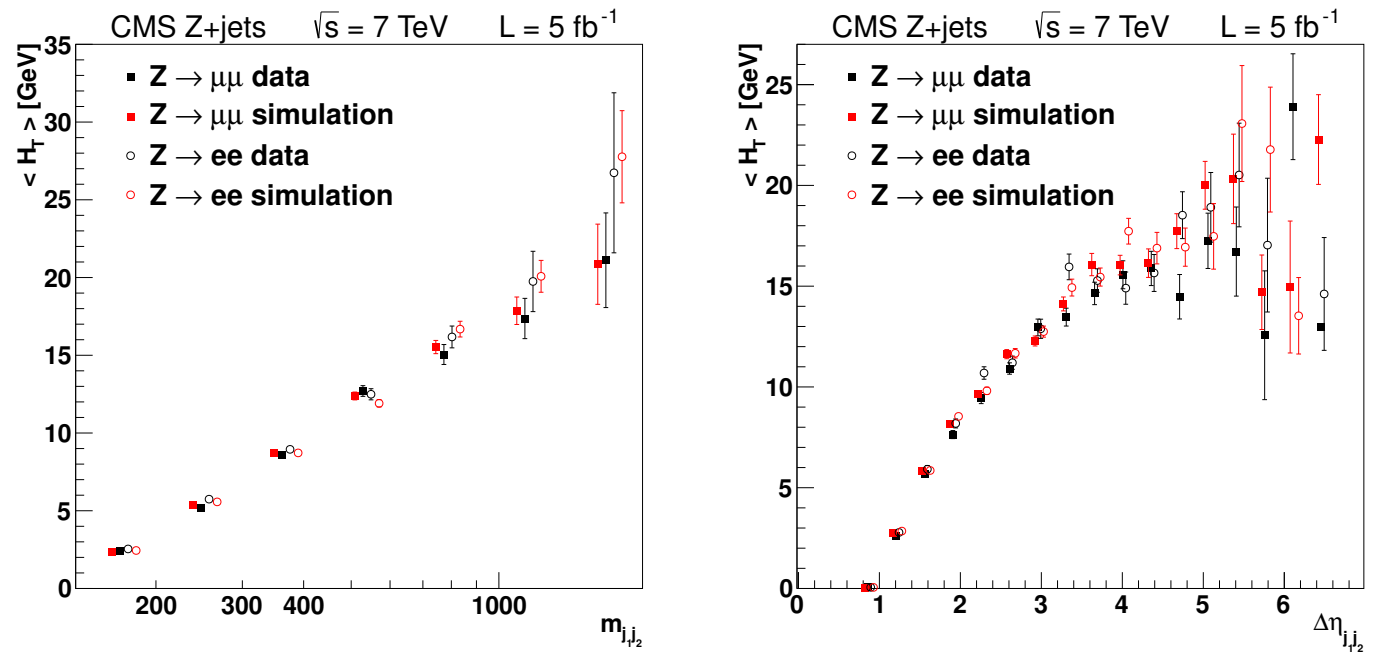

Figure 7. Average $H_{\mathrm{T}}$ of the three leading soft track jets in the pseudorapidity gap between the tagging jets for $p_{\mathrm{T}}^{\mathrm{j}_{1}, \mathrm{j}_{2}}>65,40 \mathrm{GeV}$ as a function of the dijet invariant mass (left) and the dijet $\Delta \eta_{\mathrm{j}_{1} \mathrm{j}_{2}}$ separation (right) for both the dielectron and dimuon channels in DY Zjj events. The data points and the points from simulation are shown with the statistical uncertainties.

- the difference in the pseudorapidity, $\Delta \eta_{\mathrm{j}_{1} \mathrm{j}_{2}}$, between the two most forward-backward jets (which are not necessarily the two highest $-p_{\mathrm{T}}$ jets);

- the cosine of the azimuthal angle difference, $\cos \left|\phi_{\mathrm{j}_{1}}-\phi_{\mathrm{j}_{2}}\right|=\cos \Delta \phi_{\mathrm{j}_{1} \mathrm{j}_{2}}$, between the two most forward-backward jets.

These observables are investigated following the prescriptions and suggestions in ref. [40], where the model dependence is estimated by comparing the predictions from MCFM [29], PYTHIA, ALPGEN [41]+PYTHIA, and the HEJ [42] programs.

The observables $N_{j}, H_{\mathrm{T}}, \Delta \eta_{\mathrm{j}_{1} j_{2}}$, and $\cos \Delta \phi_{\mathrm{j}_{1} \mathrm{j}_{2}}$ are measured for jets with $p_{\mathrm{T}}>40 \mathrm{GeV}$. The events are required to satisfy the $\mathrm{Z}_{\mu \mu}$ and $\mathrm{Z}_{\mathrm{ee}}$ selection criteria. Figures 8 and 9 show the average number of jets and the average $\cos \Delta \phi_{\mathrm{j}_{1} \mathrm{j}_{2}}$ as a function of the total $H_{\mathrm{T}}$ and $\Delta \eta_{\mathrm{j}_{1} \mathrm{j}_{2}}$. The MADGRAPH + PYTHIA (ME-PS) predictions are in reasonable agreement with the data.

\section{Signal cross section measurement}

\subsection{Signal extraction using the dijet mass fit}

The signal cross section in the $\mu^{+} \mu^{-}$channel is extracted from a fit of the $m_{\mathrm{j}_{1} \mathrm{j}_{2}}$ data distribution obtained after the $\mathrm{Z}_{\mu \mu}$ selection and requirements TJ1 and YZ described in section 3. The distribution is fitted to the DY $\mu \mu \mathrm{jj}$ background and the EW $\mu \mu \mathrm{jj}$ signal processes with MC templates. Figure 10 shows the $m_{\mathrm{j}_{1} \mathrm{j}_{2}}$ distribution where the expected contributions from the dominant DY $\mu \mu \mathrm{jj}$ background and the EW $\mu \mu \mathrm{jj}$ signal are evaluated from the fit, while the contributions from the small t $\bar{t}$ and diboson backgrounds are estimated from simulation. 

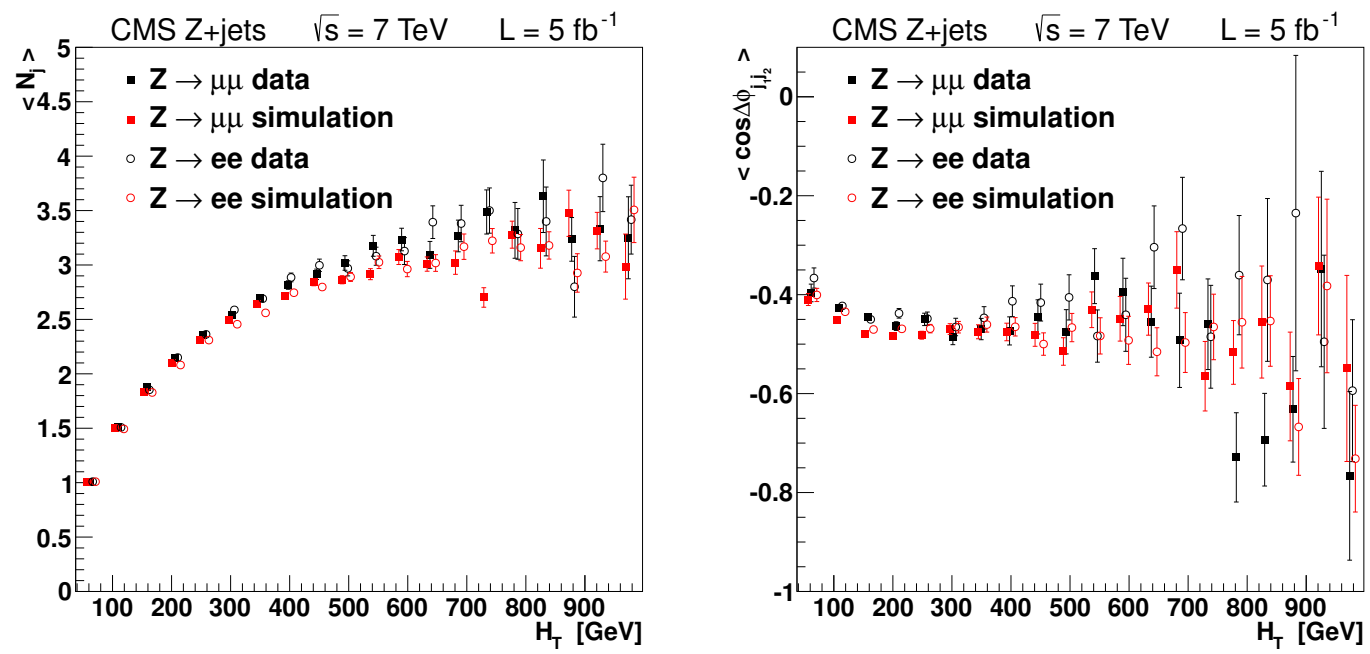

Figure 8. Average number of jets with $p_{\mathrm{T}}>40 \mathrm{GeV}$ as a function of the their total $H_{\mathrm{T}}$ in $\mathrm{Z}$ plus at least one jet events (left) and average $\cos \Delta \phi_{\mathrm{j}_{1} \mathrm{j}_{2}}$ as a function of the total $H_{\mathrm{T}}$ in DY Zjj events (right). The data points and the points from simulation are shown with the statistical uncertainties.
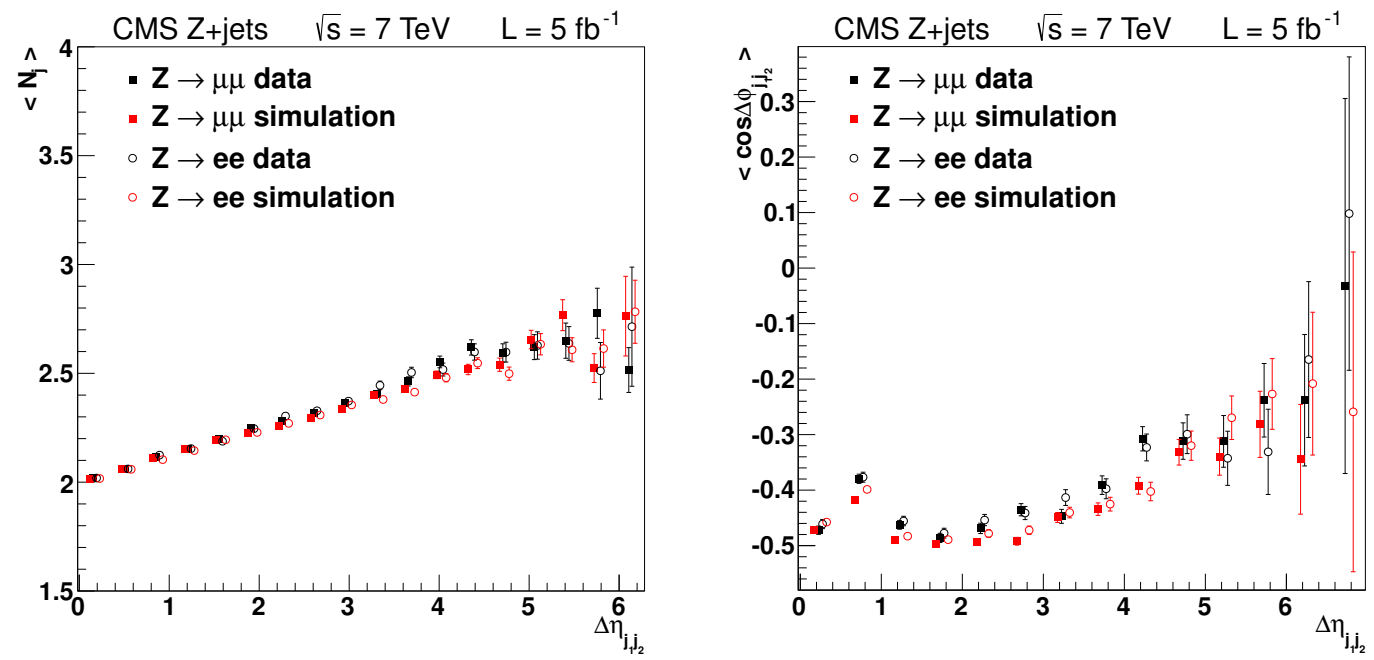

Figure 9. Average number of jets with $p_{\mathrm{T}}>40 \mathrm{GeV}$ as a function of $\Delta \eta_{\mathrm{j}_{1} \mathrm{j}_{2}}$ (left) and average $\cos \Delta \phi_{\mathrm{j}_{1} \mathrm{j}_{2}}$ as a function of $\Delta \eta_{\mathrm{j}_{1} \mathrm{j}_{2}}$ separation (right) in DY Z $\mathrm{jj}$ events. The data points and the points from simulation are shown with the statistical uncertainties.

A likelihood fit with Poisson statistics is performed following the procedure [43] using the TFractionFitter method in Rоот [44]. The free parameters of the fit, $s$ and $b$, are the ratios of the measured to the expected event yields of the EW $\mu \mu \mathrm{jj}$ signal and the DY $\mu \mu \mathrm{jj}$ background. The number of expected events is computed in the kinematical region defined in section 2. The numbers of the $t \bar{t}$ and diboson background events expected from simulation are fixed in the fit. The fit yields $s=1.14 \pm 0.28$ (stat.), $b=0.869 \pm 0.008$ (stat.) for JPT jets, and $s=1.14 \pm 0.30$ (stat.), $b=0.897 \pm 0.008$ (stat.) for PF jets. The systematic uncertainties of $s$ are discussed in section 6.2. 


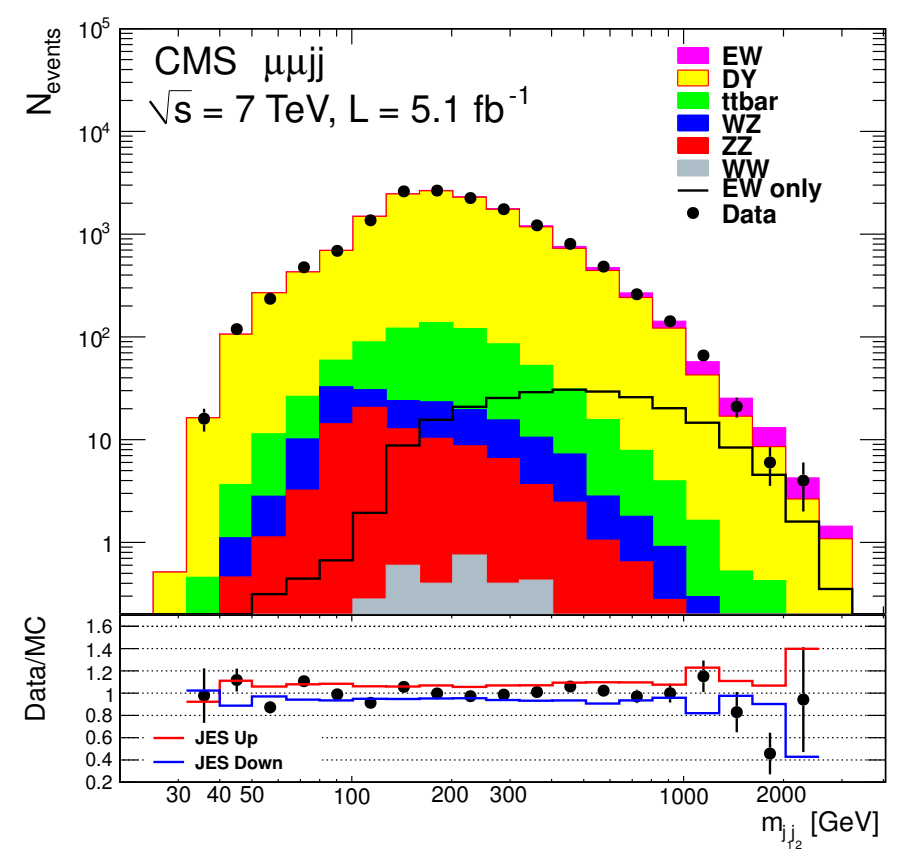

Figure 10. The $m_{\mathrm{j}_{1} \mathrm{j}_{2}}$ distribution after the $\mathrm{Z}_{\mu \mu}$, TJ1, and $\mathrm{YZ}$ selections (see section 3 ). The expected contributions from the dominant DY $\mu \mu \mathrm{jj}$ background and the EW $\mu \mu \mathrm{jj}$ signal processes are evaluated from a fit, while the contributions from the small $t \bar{t}$ and diboson backgrounds are estimated from simulation. The solid line with the label "EW only" shows the $m_{\mathrm{j}_{1} \mathrm{j}_{2}}$ distribution for the signal alone. The bottom panel shows the ratio of data over the expected contribution of the signal plus background. The region between two lines, with the labels JES Up and JES Down, shows the $1 \sigma$ uncertainty due to the jet energy scale uncertainty. The data points are shown with the statistical uncertainties.

\subsection{Systematic uncertainties}

The sources and the absolute values of the systematic uncertainties on the estimated signal value of $s$ are described below and summarized in table 6 .

The following effects are taken into account in the extraction of the signal cross section from the fit of the $m_{\mathrm{j}_{1} \mathrm{j}_{2}}$ distribution:

- The theoretical uncertainty on the $m_{\mathrm{j}_{1} \mathrm{j}_{2}}$ shape for the dominant DY $\mu \mu \mathrm{jj}$ background process. The $m_{\mathrm{j}_{1} \mathrm{j}_{2}}$ shape given by the NLO calculation of MCFM is used to correct the shape of MADGRAPH with jets built from partons and propagated to the reconstructed dijet mass with a procedure that matches the reconstructed and the parton jets. The fit is then repeated with the modified shape. The systematic uncertainty is taken as $s_{\mathrm{NLO}}-s_{\mathrm{MADGRAPH}}$, where $s_{\mathrm{NLO}}$ and $s_{\mathrm{MADGRAPH}}$ are the values of the parameter $s$ extracted from the fit of the $m_{\mathrm{j}_{1} \mathrm{j}_{2}}$ distribution given by MADGRAPH with and without corrections to the NLO shape. The uncertainty of the $m_{\mathrm{j}_{1} \mathrm{j}_{2}}$ shape at NLO due to the uncertainties in the QCD factorization and renormalization scales, $\mu_{\mathrm{F}}$ and $\mu_{\mathrm{R}}$, is much smaller than the difference between the shapes given by MADGRAPH and the NLO calculations. The QCD scale in the NLO calculations is varied from $\mu_{0} / 2$ to $2 \mu_{0}$. The $m_{\mathrm{j}_{1} \mathrm{j}_{2}}$ shape uncertainty due to the PDFs is found to be negligible. 


\begin{tabular}{|l|c|}
\hline Source of uncertainty & Uncertainty \\
\hline \multicolumn{2}{|c|}{ Theoretical uncertainties } \\
\hline Background modeling & 0.20 \\
Signal modeling & 0.05 \\
tĒ cross section & 0.02 \\
Diboson cross sections & 0.01 \\
\hline Total & 0.21 \\
\hline \multicolumn{2}{|c|}{ Experimental uncertainties } \\
\hline JES+JER & 0.44 \\
Pileup modeling & 0.05 \\
MC statistics & 0.14 \\
Dimuon selection & 0.02 \\
\hline Total & 0.47 \\
\hline Luminosity & 0.02 \\
\hline
\end{tabular}

Table 6. Sources and absolute values of the systematic uncertainties on the estimated ratio $s$ of measured over expected EW Zjj yields. The simulation of the signal includes $m_{\mathrm{jj}}>120 \mathrm{GeV}$.

- The theoretical uncertainty of the signal acceptance. The acceptance is obtained using the NLO calculation VBFNLO as well as using MAdGRAPH. Since VBFnlO does not generate events that can be passed through the detector simulation, the following parton-level requirements, similar to those used in the analysis were applied: $p_{\mathrm{T}}^{\ell}>20 \mathrm{GeV},\left|\eta^{\ell}\right|<2.4, p_{\mathrm{T}}^{\mathrm{j}}>50 \mathrm{GeV},\left|\eta^{\mathrm{j}}\right|<3.6$. The acceptance is calculated as the ratio of the cross section with parton-level selection to the cross section with the selection in the MADGRAPH simulation of the signal $\left(m_{\mathrm{jj}}>120 \mathrm{GeV}\right.$; see section 2$)$. The 5\% difference between the VBFNLO and MADGRAPH acceptances is taken as the systematic uncertainty. The $m_{\mathrm{jj}}$ shapes given by the VBFNLO program and MADGRAPH simulation are found to be very similar, and therefore the shape difference is not included in the signal modeling uncertainty. The signal acceptance used in the analysis is evaluated, however, with MADGRAPH, applying the selections as described in section 2.

- The uncertainty on the jet energy scale (JES). The $m_{\mathrm{j}_{1} \mathrm{j}_{2}}$ fit is repeated with events simulated with the jet energy varied by the JES uncertainty [19]. The difference between the values of the parameter $s$ extracted from the fit with simulated events with the adjusted jet energy is taken as the systematic uncertainty.

- The uncertainty on the jet energy resolution (JER). The $m_{\mathrm{j}_{1} \mathrm{j}_{2}}$ fit is repeated with events simulated with the correction factor varied by the JER uncertainty [19]. The difference between the values of the parameter $s$ extracted from the fit using simulated events with the adjusted data-to-simulation correction factor is taken as the systematic uncertainty. 
- The uncertainty on the pileup modeling via re-weighting of the simulated events according to the distribution of the number of interactions per beam crossing. The distribution is re-evaluated with the total inelastic cross section varied by $\pm 5 \%$ around the nominal value of $68 \mathrm{mb}$, based on a set of models consistent with the cross section measured by the CMS experiment [45].

- The uncertainty due to the limited number of events available in the simulated samples (MC statistics).

- The uncertainties on the expected yields of $t \bar{t}$ and diboson events corresponding to the theoretical cross section prediction uncertainties.

In addition, the following systematic uncertainties are included in the estimation of the cross section:

- the estimated $2.2 \%$ uncertainty on the integrated luminosity [46],

- the $1 \%$ uncertainty on the data-to-simulation correction factor for the efficiency of the lepton reconstruction, identification, isolation, and trigger, which is measured with $\mathrm{Z} \rightarrow \ell \ell$ events.

\subsection{Signal extraction using MVA analysis}

The signal is extracted with multivariate analyses in the $\mu^{+} \mu^{-}$and $\mathrm{e}^{+} \mathrm{e}^{-}$channels. The events are required to pass the $\mathrm{Z}_{\mu \mu}$ or $\mathrm{Z}_{\text {ee }}$ selection criteria and the tagging jet requirement TJ1. A boosted decision tree with decorrelation (BDTD option in the TMVA package [47]) is trained to give a high output value for signal-like events based on the following observables

- $p_{\mathrm{T}}^{\mathrm{j}_{1}}, p_{\mathrm{T}}^{\mathrm{j}_{2}}, m_{\mathrm{j}_{1} \mathrm{j}_{2}}, \Delta \eta_{\mathrm{j}_{1} \mathrm{j}_{2}}$, and $y^{*}$ variables as defined in section 3 ;

- $p_{\mathrm{T}}^{\ell \ell}$ : the $p_{\mathrm{T}}$ of the dilepton system;

- $y_{\ell \ell}$ : the rapidity of the dilepton system;

- $\eta_{\mathrm{j} 1}+\eta_{\mathrm{j} 2}$ : the sum of the pseudorapidities of the two tagging jets;

- $\Delta \phi_{\mathrm{j}_{1} \mathrm{j}_{2}}$ : the azimuthal separation of the two tagging jets;

- $\Delta \phi\left(\ell \ell, \mathrm{j}_{1}\right)$ and $\Delta \phi\left(\ell \ell, \mathrm{j}_{2}\right)$ : the azimuthal separations between the dilepton system and the two tagging jets.

In the $\mathrm{e}^{+} \mathrm{e}^{-}$channel the gluon-quark likelihood values for the tagging jets are also used as inputs. In the DY $\ell \ell \mathrm{jj}$ background about $50 \%$ of the jets originate from gluons while in the EW $\ell \ell \mathrm{jj}$ signal process the tagging jets are only initiated by quarks. A likelihood discriminator separates the gluon-originated jets from the quark-originated jets. The discriminator makes use of five internal jet properties, built from the jet constituents. These are related to the two angular spreads (root mean square) of the constituents in the $\eta$ $\phi$ plane, the asymmetry (pull) of the constituents with respect to the center of the jet, 

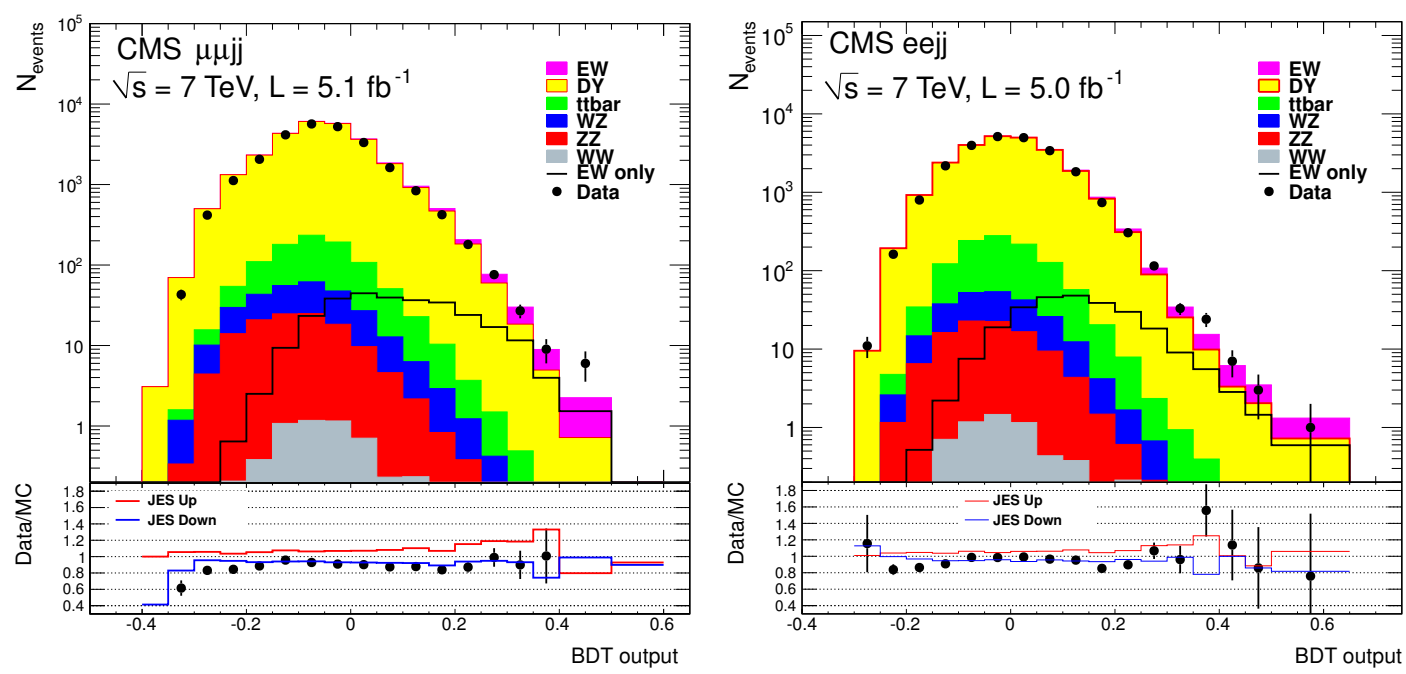

Figure 11. The BDT output distributions for the $\mu^{+} \mu^{-}$channel (left) obtained with JPT jets and for the $\mathrm{e}^{+} \mathrm{e}^{-}$channel (right) obtained with PF jets after applying the $\mathrm{Z}_{\mu \mu}$ and $\mathrm{Z}_{\mathrm{ee}}$ selection criteria, respectively, with the tagging jet requirement TJ1. The expected contributions from the signal and background processes are evaluated from simulation. The solid line with the label "EW only" shows the BDT output distribution for the signal alone. The bottom panels show the ratio of data over the expected contribution of the signal plus background. The region between the two lines, with the labels JES Up and JES Down, shows the $1 \sigma$ uncertainty of the simulation prediction due to the jet energy scale uncertainty. The data points are shown with the statistical uncertainties.

the multiplicity of the constituents, and the maximum energy fraction carried by a single constituent. The validations of the five input variables and of the gluon-quark likelihood output have been carried out using the multijet, $\mathrm{Z}+$ jet, and photon + jet samples, for which the relative differences between data and simulation are within 10\%. To assess the systematic uncertainty from the usage of this tool, the gluon likelihood output in the simulated samples has been modified in accord with the differences observed in the three samples. The use of the gluon-quark likelihood discriminator leads to a decrease of the statistical uncertainty of the measured signal in the $\mathrm{e}^{+} \mathrm{e}^{-}$channel by $5 \%$.

The BDT is trained with EW $\ell \ell j \mathrm{j}$ simulated events for the signal model along with the DY $\ell \ell j j$ and $t \bar{t}$ simulated events for the background model. The BDT output value is proportional to the probability that the event belongs to the signal: the higher the value, the higher the probability. The BDT output distributions for the two lepton modes from various production mechanisms are shown in figure 11 where the expected contributions from the signal and background processes are evaluated from simulation.

The signal cross section is extracted from the fit of the BDT output distributions for data with the method described in section 6.1, for the $m_{\mathrm{j}_{1} \mathrm{j}_{2}}$ distributions. For the $\mu^{+} \mu^{-}$ channel, the best fits are $s=0.90 \pm 0.19$ (stat.), $b=0.905 \pm 0.006$ (stat.) with JPT jets and $s=0.85 \pm 0.18$ (stat.), $b=0.937 \pm 0.007$ (stat.) with PF jets. For the $\mathrm{e}^{+} \mathrm{e}^{-}$channel, with PF jets, the best fit is $s=1.17 \pm 0.27$ (stat.), $b=0.957 \pm 0.010$ (stat.). The value of the parameter $b$ obtained from the fit is below unity by $5-10 \%$. It is however consistent with unity within the JES uncertainty and the systematic uncertainty in the MADGRAPH simulation of the DY $\ell$ ljj process as discussed in section 3. 
Figure 12 shows the BDT output distributions for the $\mu^{+} \mu^{-}$(left) and $\mathrm{e}^{+} \mathrm{e}^{-}$(right) channels, where the expected contributions from the dominant DY $\ell \ell j j$ background and the EW $\ell$ ljj signal processes are evaluated from the fit; the contributions from the small $\mathrm{t} \overline{\mathrm{t}}$ and diboson backgrounds are taken from the simulation estimates.

The presence of the signal is clearly seen at high values of the BDT output $(>0.25)$ for both dimuon and dielectron channels, and in the cases when the dominant DY $\ell \ell j \mathrm{j}$ background is evaluated from simulation (figure 11) or from the fit (figure 12).

In figure 12 the bottom panels show the significance observed in data (histogram) and expected from simulation (solid purple line), while the dashed blue line shows the background modeling uncertainty. The observed signal significance in bin $i$ of the BDT output distribution is calculated as

$$
S_{i}^{\text {observed }}=\frac{N_{i}^{\text {data }}-N_{i}^{\mathrm{bkg}}}{\sqrt{N_{i}^{\mathrm{bkg}}+\left(\Delta B_{i}^{\mathrm{JES}}\right)^{2}}},
$$

where $N_{i}^{\text {data }}$ and $N_{i}^{\text {bkg }}$ are the number of the observed events and the number of the simulated background events obtained from the fit, respectively. $\Delta B_{i}^{\mathrm{JES}}$ is the dominant experimental systematic uncertainty due to the JES, calculated as

$$
\Delta B_{i}^{\mathrm{JES}}=\sqrt{0.5\left[\left(N_{i}^{\mathrm{bkg}}-N_{i, \mathrm{JESup}}^{\mathrm{bkg}}\right)^{2}+\left(N_{i}^{\mathrm{bkg}}-N_{i, \mathrm{JESdn}}^{\mathrm{bkg}}\right)^{2}\right]},
$$

where $N_{i, \mathrm{JESup}}^{\mathrm{bkg}}$ and $N_{i, \mathrm{JESdn}}^{\mathrm{bkg}}$ are the numbers of the simulated background events from the fit with the jet energy varied by the JES uncertainty. The expected signal significance is calculated as

$$
S_{i}^{\text {expected }}=\frac{N_{i}^{\mathrm{EW} \mathrm{Zjj}}}{\sqrt{N_{i}^{\mathrm{bkg}}+\left(\Delta B_{i}^{\mathrm{JES}}\right)^{2}}},
$$

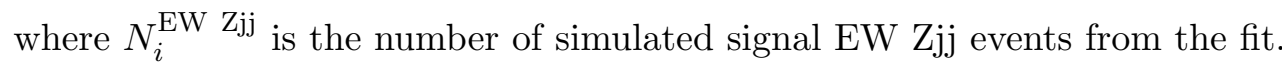

The background modeling uncertainty is calculated as

$$
\left(N_{i}^{\mathrm{MCFM}}-N_{i}^{\mathrm{bkg}}\right) / \sqrt{N_{i}^{\mathrm{bkg}}+\left(\Delta B_{i}^{\mathrm{JES}}\right)^{2}},
$$

where $N_{i}^{\mathrm{MCFM}}$ is the number of the simulated background events obtained from a new fit. The fit uses a modified BDT output distribution for the DY $\ell \ell j \mathrm{j}$ process. This distribution is evaluated using the $m_{\mathrm{j}_{1} \mathrm{j}_{2}}$ shape obtained from the NLO calculation of MCFM, as explained in section 6.2.

The sources of the systematic uncertainties on the estimated signal value of $s$ are those discussed in section 6.2. The absolute values of the systematic uncertainties on the value of $s$ for the BDT analysis are shown in table 7 for the $\mu^{+} \mu^{-}$and $\mathrm{e}^{+} \mathrm{e}^{-}$modes. The uncertainties are smaller than those from the $m_{\mathrm{j}_{1} \mathrm{j}_{2}}$ fit analysis since the BDT approach provides better separation between signal and background.

The BDT analysis in the $\mu^{+} \mu^{-}$channel is repeated for events passing the additional requirement of $\left|y^{*}\right|<1.2$, as used in the $m_{\mathrm{j}_{1} \mathrm{j}_{2}}$ analysis. In this case the best fit values 

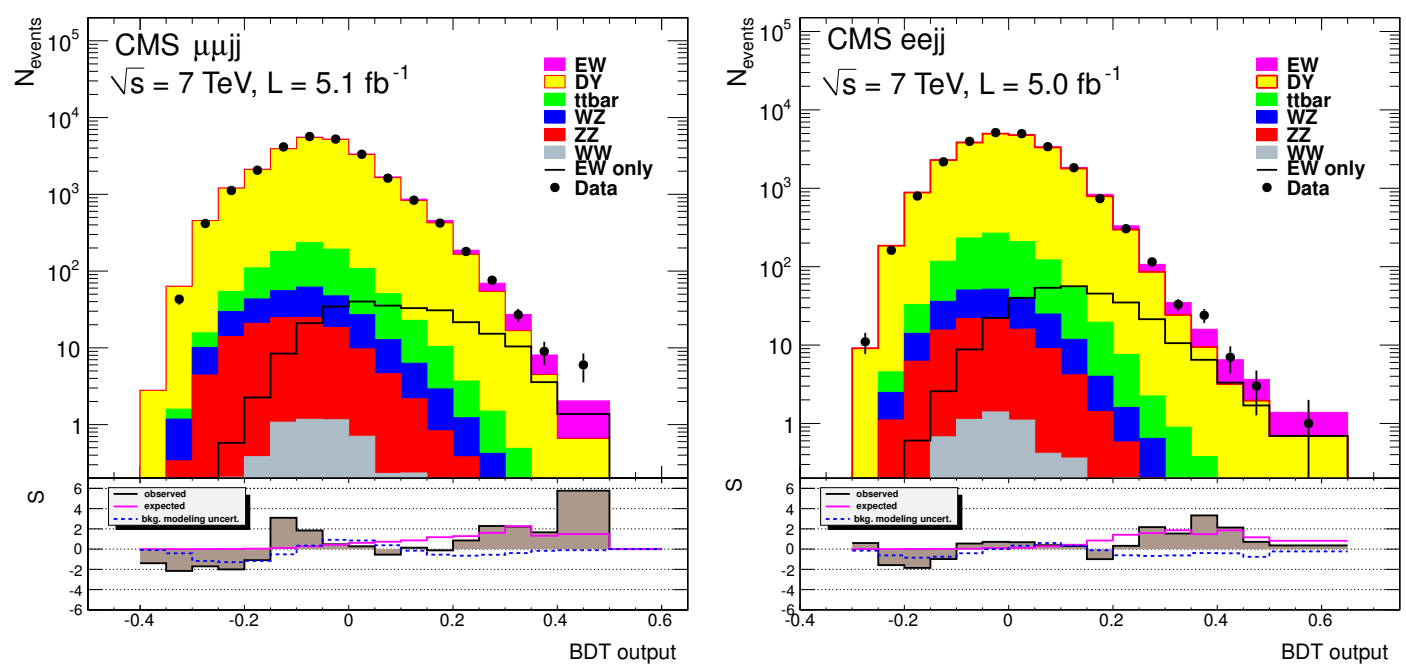

Figure 12. The BDT output distributions for the $\mu^{+} \mu^{-}$channel (left) obtained with JPT jets and for the $\mathrm{e}^{+} \mathrm{e}^{-}$channel (right) obtained with $\mathrm{PF}$ jets after the respective $\mathrm{Z}_{\mu \mu}$ and $\mathrm{Z}_{\mathrm{ee}}$ selections and the tagging jet requirement TJ1. The expected contributions from the dominant DY $\ell \ell j \mathrm{j}$ background and the EW $\ell$ jjj signal processes are evaluated from the fit. The contributions from the small $t \bar{t}$ and diboson backgrounds are estimated from simulation. The solid line with the label "EW only" shows the BDT output distribution for the signal alone. The bottom panels show the significance observed in data (histogram) and expected from simulation (solid purple line). The dashed blue line shows the background modeling uncertainty. The calculation of the significance and background modeling uncertainty are explained in the text. The data points are shown with the statistical uncertainties.

are: $s=1.50 \pm 0.26$ (stat.), $b=0.863 \pm 0.007$ (stat.) for the analysis with JPT jets and $s=1.37 \pm 0.25$ (stat.), $b=0.862 \pm 0.007$ (stat.) for the analysis with PF jets. These values are compatible with those obtained from the method based on the $m_{\mathrm{j}_{1} \mathrm{j}_{2}}$ fit, as described in section 6.1 .

\subsection{Results}

The presence of the signal is confirmed in the dimuon and dielectron channels by using two alternative jet reconstruction algorithms and two methods of signal extraction.

The BDT analysis provides smaller uncertainties on the parameter $s$, and therefore the result is based on this analysis. The measured cross section is $\sigma_{\text {meas }}=s \times$ $\sigma_{\text {MAdGraph }}(E W \ell \ell \mathrm{jj})$, where $\sigma_{\mathrm{MADGRAPH}}(\mathrm{EW} \ell \ell \mathrm{jj})=162 \mathrm{fb}$ per lepton flavor is the cross section obtained from the MADGRAPH simulation using CTEQ6L1 [28].

The signal cross section given by MADGRAPH is obtained for event generation with the following selections at the parton level: $m_{\ell \ell}>50 \mathrm{GeV}, p_{\mathrm{T}}^{\mathrm{j}}>25 \mathrm{GeV},\left|\eta^{\mathrm{j}}\right|<4.0$, $m_{\mathrm{jj}}>120 \mathrm{GeV}$. The parton-level requirements on the jet $p_{\mathrm{T}}$ and $\eta$ maximize the signal selection efficiency relative to the actual selection applied to the data, while keeping the fraction of the events which fail the parton-level requirements but pass the data selection criteria at a negligible level. 


\begin{tabular}{|l|c|c|}
\hline \multirow{2}{*}{ Source of uncertainty } & \multicolumn{2}{|c|}{ Uncertainty } \\
\cline { 2 - 3 } & $\mu^{+} \mu^{-}$channel & $\mathrm{e}^{+} \mathrm{e}^{-}$channel \\
\hline \multicolumn{2}{|c|}{ Theoretical uncertainties } \\
\hline Background modeling & 0.15 & 0.16 \\
Signal modeling & 0.05 & 0.05 \\
$\mathrm{t} \overline{\mathrm{t}}$ cross section & 0.03 & 0.03 \\
Diboson cross sections & 0.02 & 0.02 \\
\hline Total Experimental uncertainties \\
\hline \multicolumn{2}{|c|}{0.17} \\
\hline JES+JER & 0.22 & 0.29 \\
Pileup modeling & 0.03 & 0.03 \\
MC statistics & 0.13 & 0.19 \\
Gluon-quark discriminator & not used & 0.02 \\
Dilepton selection & 0.02 & 0.02 \\
\hline Total & 0.26 & 0.35 \\
\hline Luminosity & 0.02 & 0.03 \\
\hline
\end{tabular}

Table 7. The sources and absolute values of systematic uncertainties on the estimated ratio $s$ of measured over expected EW Zjj yields in the BDT analysis for the $\mu^{+} \mu^{-}$and $\mathrm{e}^{+} \mathrm{e}^{-}$channels.

The cross section for the dimuon mode with JPT jets is:

$$
\sigma_{\mu \mu}^{\mathrm{EW}}(\mathrm{JPT})=146 \pm 31 \text { (stat.) } \pm 42 \text { (exp. syst.) } \pm 26 \text { (th. syst.) } \pm 3 \text { (lum.) fb. }
$$

The cross section for the dimuon mode with PF jets is

$$
\sigma_{\mu \mu}^{\mathrm{EW}}(\mathrm{PF})=138 \pm 29 \text { (stat.) } \pm 40 \text { (exp. syst.) } \pm 25 \text { (th. syst.) } \pm 3 \text { (lum.) fb. }
$$

The measurements for the dimuon mode, with the two different jet reconstruction algorithms, are compatible.

In the dielectron mode with PF jets, the cross section is:

$$
\sigma_{\mathrm{ee}}^{\mathrm{EW}}(\mathrm{PF})=190 \pm 44 \text { (stat.) } \pm 57 \text { (exp. syst.) } \pm 27 \text { (th. syst.) } \pm 4 \text { (lum.) fb. }
$$

The measured cross sections agree with the theoretical value of $\sigma_{\mathrm{VBFNLO}}(\mathrm{EW} \ell \ell \mathrm{jj})=166 \mathrm{fb}$, calculated with next-to-leading order QCD corrections using the same parton level selections as those applied in the signal event generation by MADGraPH. The cross sections obtained in the $\mu^{+} \mu^{-}$and $\mathrm{e}^{+} \mathrm{e}^{-}$analyses using PF jets is combined and the average cross section is:

$$
\sigma_{\ell \ell(\ell=\mathrm{e}, \mu)}^{\mathrm{EW}}=154 \pm 24 \text { (stat.) } \pm 46 \text { (exp. syst.) } \pm 27 \text { (th. syst.) } \pm 3 \text { (lum.) fb. }
$$




\section{Summary}

A measurement of the electroweak production of a $\mathrm{Z}$ boson in association with two jets in pp collisions at $\sqrt{s}=7 \mathrm{TeV}$ has been carried out with the CMS detector using an integrated luminosity of $5 \mathrm{fb}^{-1}$. The cross section for the EW $\ell \ell \mathrm{jj}(\ell=\mathrm{e}, \mu)$ production process, with $m_{\ell \ell}>50 \mathrm{GeV}, p_{\mathrm{T}}^{\mathrm{j}}>25 \mathrm{GeV},\left|\eta^{\mathrm{j}}\right|<4.0, m_{\mathrm{jj}}>120 \mathrm{GeV}$, is $\sigma=154 \pm$ 24 (stat.) \pm 46 (exp. syst.) \pm 27 (th. syst.) \pm 3 (lum.) fb. The measurement is in agreement with the theoretical cross section of $166 \mathrm{fb}$, obtained with calculations including nextto-leading order QCD corrections based on the CT10 [34] parton distribution functions. A significance of 2.6 standard deviations has been obtained for the observation of EW production of the $\mathrm{Z}$ boson with two tagging jets. The measured hadronic activity in events with Drell-Yan production in association with two jets is in good agreement with simulation. This is the first measurement of EW production of a $\mathrm{Z}$ boson with two jets at a hadron collider, and constitutes an important foundation for the more general study of vector boson fusion processes, of relevance for Higgs boson searches and for measurements of electroweak gauge couplings and vector-boson scattering.

\section{Acknowledgments}

We congratulate our colleagues in the CERN accelerator departments for the excellent performance of the LHC and thank the technical and administrative staffs at CERN and at other CMS institutes for their contributions to the success of the CMS effort. In addition, we gratefully acknowledge the computing centers and personnel of the Worldwide LHC Computing Grid for delivering so effectively the computing infrastructure essential to our analyses. Finally, we acknowledge the enduring support for the construction and operation of the LHC and the CMS detector provided by the following funding agencies: BMWF and FWF (Austria); FNRS and FWO (Belgium); CNPq, CAPES, FAPERJ, and FAPESP (Brazil); MEYS (Bulgaria); CERN; CAS, MoST, and NSFC (China); COLCIENCIAS (Colombia); MSES (Croatia); RPF (Cyprus); MoER, SF0690030s09 and ERDF (Estonia); Academy of Finland, MEC, and HIP (Finland); CEA and CNRS/IN2P3 (France); BMBF, DFG, and HGF (Germany); GSRT (Greece); OTKA and NKTH (Hungary); DAE and DST (India); IPM (Iran); SFI (Ireland); INFN (Italy); NRF and WCU (Republic of Korea); LAS (Lithuania); CINVESTAV, CONACYT, SEP, and UASLP-FAI (Mexico); MSI (New Zealand); PAEC (Pakistan); MSHE and NSC (Poland); FCT (Portugal); JINR (Armenia, Belarus, Georgia, Ukraine, Uzbekistan); MON, RosAtom, RAS and RFBR (Russia); MSTD (Serbia); SEIDI and CPAN (Spain); Swiss Funding Agencies (Switzerland); NSC (Taipei); ThEPCenter, IPST and NSTDA (Thailand); TUBITAK and TAEK (Turkey); NASU (Ukraine); STFC (United Kingdom); DOE and NSF (USA).

Individuals have received support from the Marie-Curie programme and the European Research Council and EPLANET (European Union); the Leventis Foundation; the A. P. Sloan Foundation; the Alexander von Humboldt Foundation; the Belgian Federal Science Policy Office; the Fonds pour la Formation à la Recherche dans l'Industrie et dans l'Agriculture (FRIA-Belgium); the Agentschap voor Innovatie door Wetenschap en Tech- 
nologie (IWT-Belgium); the Ministry of Education, Youth and Sports (MEYS) of Czech Republic; the Council of Science and Industrial Research, India; the Compagnia di San Paolo (Torino); the HOMING PLUS programme of Foundation for Polish Science, cofinanced by EU, Regional Development Fund; and the Thalis and Aristeia programmes cofinanced by EU-ESF and the Greek NSRF.

Open Access. This article is distributed under the terms of the Creative Commons Attribution License which permits any use, distribution and reproduction in any medium, provided the original author(s) and source are credited.

\section{References}

[1] C. Oleari and D. Zeppenfeld, Next-to-leading order QCD corrections to $W$ and $Z$ production via vector-boson fusion, Phys. Rev. D 69 (2004) 093004 [hep-ph/0310156] [INSPIRE].

[2] D.L. Rainwater, R. Szalapski and D. Zeppenfeld, Probing color singlet exchange in $Z+$ two jet events at the CERN LHC, Phys. Rev. D 54 (1996) 6680 [hep-ph/9605444] [INSPIRE].

[3] V.A. Khoze, M.G. Ryskin, W.J. Stirling and P.H. Williams, A Z monitor to calibrate Higgs production via vector boson fusion with rapidity gaps at the LHC, Eur. Phys. J. C 26 (2003) 429 [hep-ph/0207365] [INSPIRE].

[4] U. Baur and D. Zeppenfeld, Measuring three vector boson couplings in $q q \rightarrow q q W$ at the SSC, hep-ph/9309227 [INSPIRE].

[5] D.L. Rainwater, D. Zeppenfeld and K. Hagiwara, Searching for $H \rightarrow \tau^{+} \tau^{-}$in weak boson fusion at the CERN LHC, Phys. Rev. D 59 (1998) 014037 [hep-ph/9808468] [INSPIRE].

[6] T. Plehn, D.L. Rainwater and D. Zeppenfeld, A method for identifying $H \rightarrow \tau^{+} \tau^{-} \rightarrow e^{ \pm} \mu^{\mp} p_{T}$ at the CERN LHC, Phys. Rev. D 61 (2000) 093005 [hep-ph/9911385] [INSPIRE].

[7] D.L. Rainwater and D. Zeppenfeld, Observing $H \rightarrow W^{*} W^{*} \rightarrow e^{ \pm} \mu^{\mp} p_{\mathrm{T}}$ in weak boson fusion with dual forward jet tagging at the CERN LHC, Phys. Rev. D 60 (1999) 113004 [Erratum ibid. D 61 (2000) 099901] [hep-ph/9906218] [INSPIRE].

[8] N. Kauer, T. Plehn, D.L. Rainwater and D. Zeppenfeld, $H \rightarrow W^{+} W^{-}$as the discovery mode for a light Higgs boson, Phys. Lett. B 503 (2001) 113 [hep-ph/0012351] [INSPIRE].

[9] ATLAS collaboration, ATLAS detector and physics performance: technical design report 2, CERN-LHCC-99-015 (1999).

[10] CMS collaboration, CMS physics technical design report, volume II: physics performance, J. Phys. G 34 (2006) 995.

[11] D. Zeppenfeld, R. Kinnunen, A. Nikitenko and E. Richter-Was, Measuring Higgs boson couplings at the CERN LHC, Phys. Rev. D 62 (2000) 013009 [hep-ph/0002036] [inSPIRE].

[12] A. Belyaev and L. Reina, $p p \rightarrow t \bar{t} H, H \rightarrow \tau^{+} \tau^{-}$: toward a model independent determination of the Higgs boson couplings at the LHC, JHEP 08 (2002) 041 [hep-ph/0205270] [INSPIRE].

[13] M. Dührssen et al., Extracting Higgs boson couplings from CERN LHC data, Phys. Rev. D 70 (2004) 113009 [hep-ph/0406323] [INSPIRE]. 
[14] ATLAS collaboration, Observation of a new particle in the search for the standard model Higgs boson with the ATLAS detector at the LHC, Phys. Lett. B 716 (2012) 1 [arXiv: 1207.7214] [INSPIRE].

[15] CMS collaboration, Observation of a new boson at a mass of $125 \mathrm{GeV}$ with the CMS experiment at the LHC, Phys. Lett. B 716 (2012) 30 [arXiv:1207.7235] [INSPIRE].

[16] CMS collaboration, The CMS experiment at the CERN LHC, 2008 JINST 3 S08004 [INSPIRE].

[17] CMS collaboration, Performance of CMS muon reconstruction in pp collision events at $\sqrt{s}=7 \mathrm{TeV}, 2012$ JINST 7 P10002 [arXiv:1206.4071] [INSPIRE].

[18] CMS collaboration, Electron reconstruction and identification at $\sqrt{s}=7 \mathrm{TeV}$, CMS-PAS-EGM-10-004 (2010).

[19] CMS collaboration, Determination of jet energy calibration and transverse momentum resolution in CMS, 2011 JINST 6 P11002 [arXiv:1107.4277] [INSPIRE].

[20] CMS collaboration, The jet plus tracks algorithm for calorimeter jet energy corrections in CMS, CMS-PAS-JME-09-002 (2009).

[21] M. Cacciari and G.P. Salam, Dispelling the $N^{3}$ myth for the $k_{t}$ jet-finder, Phys. Lett. B 641 (2006) 57 [hep-ph/0512210] [INSPIRE].

[22] M. Cacciari, G.P. Salam and G. Soyez, The anti- $k_{t}$ jet clustering algorithm, JHEP 04 (2008) 063 [arXiv: 0802.1189] [INSPIRE].

[23] CMS collaboration, Particle-flow event reconstruction in CMS and performance for jets, taus and MET, CMS-PAS-PFT-09-001 (2009).

[24] CMS collaboration, Commissioning of the particle-flow reconstruction in minimum-bias and jet events from pp collisions at $7 \mathrm{TeV}$, CMS-PAS-PFT-10-002 (2010).

[25] F. Maltoni and T. Stelzer, MadEvent: automatic event generation with MadGraph, JHEP 02 (2003) 027 [hep-ph/0208156] [INSPIRE].

[26] J. Alwall, M. Herquet, F. Maltoni, O. Mattelaer and T. Stelzer, MadGraph 5: going beyond, JHEP 06 (2011) 128 [arXiv: 1106.0522] [INSPIRE].

[27] T. Sjöstrand, S. Mrenna and P.Z. Skands, PYTHIA 6.4 physics and manual, JHEP 05 (2006) 026 [hep-ph/0603175] [INSPIRE].

[28] J. Pumplin et al., New generation of parton distributions with uncertainties from global QCD analysis, JHEP 07 (2002) 012 [hep-ph/0201195] [INSPIRE].

[29] J.M. Campbell and R. Ellis, MCFM for the Tevatron and the LHC, Nucl. Phys. Proc. Suppl. 205-206 (2010) 10 [arXiv:1007.3492] [INSPIRE].

[30] GEANT4 collaboration, S. Agostinelli et al., GEANT4: a simulation toolkit, Nucl. Instrum. Meth. A 506 (2003) 250 [inSPIRE].

[31] J. Allison et al., GEANT4 developments and applications, IEEE Trans. Nucl. Sci. 53 (2006) 270 [INSPIRE].

[32] R. Field, Min-bias and the underlying event at the LHC, Acta Phys. Polon. B 42 (2011) 2631 [arXiv: 1110.5530] [INSPIRE].

[33] K. Arnold et al., VBFNLO: a parton level Monte Carlo for processes with electroweak bosons, Comput. Phys. Commun. 180 (2009) 1661 [arXiv:0811.4559] [INSPIRE]. 
[34] H.-L. Lai et al., New parton distributions for collider physics, Phys. Rev. D 82 (2010) 074024 [arXiv: 1007.2241] [INSPIRE].

[35] T. Gleisberg et al., Event generation with SHERPA 1.1, JHEP 02 (2009) 007 [arXiv:0811.4622] [INSPIRE].

[36] CompHEP collaboration, E. Boos et al., CompHEP 4.4: automatic computations from Lagrangians to events, Nucl. Instrum. Meth. A 534 (2004) 250 [hep-ph/0403113] [INSPIRE].

[37] CMS collaboration, Tracking and primary vertex results in first $7 \mathrm{TeV}$ collisions, CMS-PAS-TRK-10-005 (2010).

[38] CMS collaboration, Commissioning of trackjets in pp collisions at $7 \mathrm{TeV}$, CMS-PAS-JME-10-006 (2010).

[39] CMS collaboration, Performance of jet reconstruction with charged tracks only, CMS-PAS-JME-08-001 (2008).

[40] SM, NLO Multileg Working Group collaboration, J. Andersen et al., The SM and NLO multileg working group: summary report, arXiv:1003.1241 [INSPIRE].

[41] M.L. Mangano, M. Moretti, F. Piccinini, R. Pittau and A.D. Polosa, ALPGEN, a generator for hard multiparton processes in hadronic collisions, JHEP 07 (2003) 001 [hep-ph/0206293] [INSPIRE].

[42] J.R. Andersen and J.M. Smillie, Multiple jets at the LHC with high energy jets, JHEP 06 (2011) 010 [arXiv: 1101.5394] [INSPIRE].

[43] R.J. Barlow and C. Beeston, Fitting using finite Monte Carlo samples, Comput. Phys. Commun. 77 (1993) 219 [INSPIRE].

[44] R. Brun and F. Rademakers, ROOT: an object oriented data analysis framework, Nucl. Instrum. Meth. A 389 (1997) 81 [inSPIRE].

[45] CMS collaboration, Measurement of the inelastic proton-proton cross section at $\sqrt{s}=7 \mathrm{TeV}$, Phys. Lett. B 722 (2013) 5 [arXiv:1210.6718] [INSPIRE].

[46] CMS collaboration, Absolute calibration of the luminosity measurement at CMS: winter 2012 update, CMS-PAS-SMP-12-008 (2012).

[47] A. Hocker, J. Stelzer, F. Tegenfeldt, H. Voss, K. Voss, et al., TMVA: toolkit for multivariate data analysis woth ROOT, PoS(ACAT) 040 [physics/0703039] [INSPIRE]. 


\section{The CMS collaboration}

\section{Yerevan Physics Institute, Yerevan, Armenia}

S. Chatrchyan, V. Khachatryan, A.M. Sirunyan, A. Tumasyan

\section{Institut für Hochenergiephysik der OeAW, Wien, Austria}

W. Adam, T. Bergauer, M. Dragicevic, J. Erö, C. Fabjan ${ }^{1}$, M. Friedl, R. Frühwirth ${ }^{1}$, V.M. Ghete, N. Hörmann, J. Hrubec, M. Jeitler ${ }^{1}$, W. Kiesenhofer, V. Knünz, M. Krammer ${ }^{1}$, I. Krätschmer, D. Liko, I. Mikulec, D. Rabady ${ }^{2}$, B. Rahbaran, C. Rohringer, H. Rohringer, R. Schöfbeck, J. Strauss, A. Taurok, W. Treberer-Treberspurg, W. Waltenberger, C.-E. Wulz ${ }^{1}$

National Centre for Particle and High Energy Physics, Minsk, Belarus

V. Mossolov, N. Shumeiko, J. Suarez Gonzalez

\section{Universiteit Antwerpen, Antwerpen, Belgium}

S. Alderweireldt, M. Bansal, S. Bansal, T. Cornelis, E.A. De Wolf, X. Janssen, A. Knutsson, S. Luyckx, L. Mucibello, S. Ochesanu, B. Roland, R. Rougny, H. Van Haevermaet, P. Van Mechelen, N. Van Remortel, A. Van Spilbeeck

\section{Vrije Universiteit Brussel, Brussel, Belgium}

F. Blekman, S. Blyweert, J. D'Hondt, A. Kalogeropoulos, J. Keaveney, M. Maes, A. Olbrechts, S. Tavernier, W. Van Doninck, P. Van Mulders, G.P. Van Onsem, I. Villella

\section{Université Libre de Bruxelles, Bruxelles, Belgium}

B. Clerbaux, G. De Lentdecker, A.P.R. Gay, T. Hreus, A. Léonard, P.E. Marage, A. Mohammadi, T. Reis, L. Thomas, C. Vander Velde, P. Vanlaer, J. Wang

\section{Ghent University, Ghent, Belgium}

V. Adler, K. Beernaert, L. Benucci, A. Cimmino, S. Costantini, S. Dildick, G. Garcia,

B. Klein, J. Lellouch, A. Marinov, J. Mccartin, A.A. Ocampo Rios, D. Ryckbosch, M. Sigamani, N. Strobbe, F. Thyssen, M. Tytgat, S. Walsh, E. Yazgan, N. Zaganidis

Université Catholique de Louvain, Louvain-la-Neuve, Belgium

S. Basegmez, G. Bruno, R. Castello, L. Ceard, C. Delaere, T. du Pree, D. Favart, L. Forthomme, A. Giammanco ${ }^{3}$, J. Hollar, V. Lemaitre, J. Liao, O. Militaru, C. Nuttens, D. Pagano, A. Pin, K. Piotrzkowski, A. Popov ${ }^{4}$, M. Selvaggi, J.M. Vizan Garcia

\section{Université de Mons, Mons, Belgium}

N. Beliy, T. Caebergs, E. Daubie, G.H. Hammad

\section{Centro Brasileiro de Pesquisas Fisicas, Rio de Janeiro, Brazil}

G.A. Alves, M. Correa Martins Junior, T. Martins, M.E. Pol, M.H.G. Souza

Universidade do Estado do Rio de Janeiro, Rio de Janeiro, Brazil

W.L. Aldá Júnior, W. Carvalho, J. Chinellato ${ }^{5}$, A. Custódio, E.M. Da Costa, D. De Jesus Damiao, C. De Oliveira Martins, S. Fonseca De Souza, H. Malbouisson, M. Malek, D. Matos Figueiredo, L. Mundim, H. Nogima, W.L. Prado Da Silva, A. Santoro, L. Soares Jorge, A. Sznajder, E.J. Tonelli Manganote ${ }^{5}$, A. Vilela Pereira 
Universidade Estadual Paulista $^{a}$, Universidade Federal do $\mathrm{ABC}^{b}$, São Paulo, Brazil

T.S. Anjos ${ }^{b}$, C.A. Bernardes ${ }^{b}$, F.A. Dias ${ }^{a}, 6$, T.R. Fernandez Perez Tomei ${ }^{a}$, E.M. Gregores ${ }^{b}$, C. Lagana ${ }^{a}$, F. Marinho ${ }^{a}$, P.G. Mercadante ${ }^{b}$, S.F. Novaes ${ }^{a}$, Sandra S. Padula ${ }^{a}$

Institute for Nuclear Research and Nuclear Energy, Sofia, Bulgaria

V. Genchev ${ }^{2}$, P. Iaydjiev², S. Piperov, M. Rodozov, S. Stoykova, G. Sultanov, V. Tcholakov,

R. Trayanov, M. Vutova

University of Sofia, Sofia, Bulgaria

A. Dimitrov, R. Hadjiiska, V. Kozhuharov, L. Litov, B. Pavlov, P. Petkov

Institute of High Energy Physics, Beijing, China

J.G. Bian, G.M. Chen, H.S. Chen, C.H. Jiang, D. Liang, S. Liang, X. Meng, J. Tao, J. Wang, X. Wang, Z. Wang, H. Xiao, M. Xu

State Key Laboratory of Nuclear Physics and Technology, Peking University, Beijing, China

C. Asawatangtrakuldee, Y. Ban, Y. Guo, Q. Li, W. Li, S. Liu, Y. Mao, S.J. Qian, D. Wang,

L. Zhang, W. Zou

Universidad de Los Andes, Bogota, Colombia

C. Avila, C.A. Carrillo Montoya, J.P. Gomez, B. Gomez Moreno, J.C. Sanabria

Technical University of Split, Split, Croatia

N. Godinovic, D. Lelas, R. Plestina ${ }^{7}$, D. Polic, I. Puljak

University of Split, Split, Croatia

Z. Antunovic, M. Kovac

Institute Rudjer Boskovic, Zagreb, Croatia

V. Brigljevic, S. Duric, K. Kadija, J. Luetic, D. Mekterovic, S. Morovic, L. Tikvica

University of Cyprus, Nicosia, Cyprus

A. Attikis, G. Mavromanolakis, J. Mousa, C. Nicolaou, F. Ptochos, P.A. Razis

Charles University, Prague, Czech Republic

M. Finger, M. Finger Jr.

Academy of Scientific Research and Technology of the Arab Republic of Egypt, Egyptian Network of High Energy Physics, Cairo, Egypt

Y. Assran ${ }^{8}$, A. Ellithi Kamel ${ }^{9}$, M.A. Mahmoud ${ }^{10}$, A. Mahrous ${ }^{11}$, A. Radi ${ }^{12,13}$

National Institute of Chemical Physics and Biophysics, Tallinn, Estonia

M. Kadastik, M. Müntel, M. Murumaa, M. Raidal, L. Rebane, A. Tiko

Department of Physics, University of Helsinki, Helsinki, Finland

P. Eerola, G. Fedi, M. Voutilainen 
Helsinki Institute of Physics, Helsinki, Finland

J. Härkönen, V. Karimäki, R. Kinnunen, M.J. Kortelainen, T. Lampén, K. Lassila-Perini,

S. Lehti, T. Lindén, P. Luukka, T. Mäenpää, T. Peltola, E. Tuominen, J. Tuominiemi,

E. Tuovinen, L. Wendland

Lappeenranta University of Technology, Lappeenranta, Finland

A. Korpela, T. Tuuva

DSM/IRFU, CEA/Saclay, Gif-sur-Yvette, France

M. Besancon, S. Choudhury, F. Couderc, M. Dejardin, D. Denegri, B. Fabbro, J.L. Faure,

F. Ferri, S. Ganjour, A. Givernaud, P. Gras, G. Hamel de Monchenault, P. Jarry, E. Locci,

J. Malcles, L. Millischer, A. Nayak, J. Rander, A. Rosowsky, M. Titov

Laboratoire Leprince-Ringuet, Ecole Polytechnique, IN2P3-CNRS, Palaiseau, France

S. Baffioni, F. Beaudette, L. Benhabib, L. Bianchini, M. Bluj ${ }^{14}$, P. Busson, C. Charlot,

N. Daci, T. Dahms, M. Dalchenko, L. Dobrzynski, A. Florent, R. Granier de Cassagnac,

M. Haguenauer, P. Miné, C. Mironov, I.N. Naranjo, M. Nguyen, C. Ochando, P. Paganini,

D. Sabes, R. Salerno, Y. Sirois, C. Veelken, A. Zabi

Institut Pluridisciplinaire Hubert Curien, Université de Strasbourg, Université de Haute Alsace Mulhouse, CNRS/IN2P3, Strasbourg, France

J.-L. Agram ${ }^{15}$, J. Andrea, D. Bloch, D. Bodin, J.-M. Brom, E.C. Chabert, C. Collard, E. Conte ${ }^{15}$, F. Drouhin ${ }^{15}$, J.-C. Fontaine ${ }^{15}$, D. Gelé, U. Goerlach, C. Goetzmann, P. Juillot, A.-C. Le Bihan, P. Van Hove

Université de Lyon, Université Claude Bernard Lyon 1, CNRS-IN2P3, Institut de Physique Nucléaire de Lyon, Villeurbanne, France

S. Beauceron, N. Beaupere, O. Bondu, G. Boudoul, S. Brochet, J. Chasserat, R. Chierici², D. Contardo, P. Depasse, H. El Mamouni, J. Fay, S. Gascon, M. Gouzevitch, B. Ille, T. Kurca, M. Lethuillier, L. Mirabito, S. Perries, L. Sgandurra, V. Sordini, Y. Tschudi, M. Vander Donckt, P. Verdier, S. Viret

Institute of High Energy Physics and Informatization, Tbilisi State University, Tbilisi, Georgia

Z. Tsamalaidze ${ }^{16}$

RWTH Aachen University, I. Physikalisches Institut, Aachen, Germany

C. Autermann, S. Beranek, B. Calpas, M. Edelhoff, L. Feld, N. Heracleous, O. Hindrichs,

K. Klein, J. Merz, A. Ostapchuk, A. Perieanu, F. Raupach, J. Sammet, S. Schael, D. Sprenger, H. Weber, B. Wittmer, V. Zhukov ${ }^{4}$

RWTH Aachen University, III. Physikalisches Institut A, Aachen, Germany M. Ata, J. Caudron, E. Dietz-Laursonn, D. Duchardt, M. Erdmann, R. Fischer, A. Güth,

T. Hebbeker, C. Heidemann, K. Hoepfner, D. Klingebiel, P. Kreuzer, M. Merschmeyer,

A. Meyer, M. Olschewski, K. Padeken, P. Papacz, H. Pieta, H. Reithler, S.A. Schmitz,

L. Sonnenschein, J. Steggemann, D. Teyssier, S. Thüer, M. Weber 
RWTH Aachen University, III. Physikalisches Institut B, Aachen, Germany

V. Cherepanov, Y. Erdogan, G. Flügge, H. Geenen, M. Geisler, W. Haj Ahmad, F. Hoehle, B. Kargoll, T. Kress, Y. Kuessel, J. Lingemann ${ }^{2}$, A. Nowack, I.M. Nugent, L. Perchalla, O. Pooth, A. Stahl

\section{Deutsches Elektronen-Synchrotron, Hamburg, Germany}

M. Aldaya Martin, I. Asin, N. Bartosik, J. Behr, W. Behrenhoff, U. Behrens, M. Bergholz ${ }^{17}$, A. Bethani, K. Borras, A. Burgmeier, A. Cakir, L. Calligaris, A. Campbell, F. Costanza, D. Dammann, C. Diez Pardos, T. Dorland, G. Eckerlin, D. Eckstein, G. Flucke, A. Geiser, I. Glushkov, P. Gunnellini, S. Habib, J. Hauk, G. Hellwig, H. Jung, M. Kasemann, P. Katsas, C. Kleinwort, H. Kluge, M. Krämer, D. Krücker, E. Kuznetsova, W. Lange, J. Leonard, K. Lipka, W. Lohmann ${ }^{17}$, B. Lutz, R. Mankel, I. Marfin, M. Marienfeld, I.-A. MelzerPellmann, A.B. Meyer, J. Mnich, A. Mussgiller, S. Naumann-Emme, O. Novgorodova, F. Nowak, J. Olzem, H. Perrey, A. Petrukhin, D. Pitzl, A. Raspereza, P.M. Ribeiro Cipriano, C. Riedl, E. Ron, M. Rosin, J. Salfeld-Nebgen, R. Schmidt ${ }^{17}$, T. SchoernerSadenius, N. Sen, M. Stein, R. Walsh, C. Wissing

\section{University of Hamburg, Hamburg, Germany}

V. Blobel, H. Enderle, J. Erfle, U. Gebbert, M. Görner, M. Gosselink, J. Haller, K. Heine, R.S. Höing, K. Kaschube, G. Kaussen, H. Kirschenmann, R. Klanner, J. Lange, T. Peiffer, N. Pietsch, D. Rathjens, C. Sander, H. Schettler, P. Schleper, E. Schlieckau, A. Schmidt, M. Schröder, T. Schum, M. Seidel, J. Sibille ${ }^{18}$, V. Sola, H. Stadie, G. Steinbrück, J. Thomsen, L. Vanelderen

\section{Institut für Experimentelle Kernphysik, Karlsruhe, Germany}

C. Barth, C. Baus, J. Berger, C. Böser, T. Chwalek, W. De Boer, A. Descroix, A. Dierlamm, M. Feindt, M. Guthoff ${ }^{2}$, C. Hackstein, F. Hartmann ${ }^{2}$, T. Hauth ${ }^{2}$, M. Heinrich, H. Held, K.H. Hoffmann, U. Husemann, I. Katkov ${ }^{4}$, J.R. Komaragiri, A. Kornmayer ${ }^{2}$, P. Lobelle Pardo, D. Martschei, S. Mueller, Th. Müller, M. Niegel, A. Nürnberg, O. Oberst, J. Ott, G. Quast, K. Rabbertz, F. Ratnikov, N. Ratnikova, S. Röcker, F.-P. Schilling, G. Schott, H.J. Simonis, F.M. Stober, D. Troendle, R. Ulrich, J. Wagner-Kuhr, S. Wayand, T. Weiler, M. Zeise

Institute of Nuclear and Particle Physics (INPP), NCSR Demokritos, Aghia Paraskevi, Greece

G. Anagnostou, G. Daskalakis, T. Geralis, S. Kesisoglou, A. Kyriakis, D. Loukas, A. Markou, C. Markou, E. Ntomari

\section{University of Athens, Athens, Greece}

L. Gouskos, T.J. Mertzimekis, A. Panagiotou, N. Saoulidou, E. Stiliaris

\section{University of Ioánnina, Ioánnina, Greece}

X. Aslanoglou, I. Evangelou, G. Flouris, C. Foudas, P. Kokkas, N. Manthos, I. Papadopoulos, E. Paradas 
KFKI Research Institute for Particle and Nuclear Physics, Budapest, Hungary

G. Bencze, C. Hajdu, P. Hidas, D. Horvath ${ }^{19}$, B. Radics, F. Sikler, V. Veszpremi, G. Vesztergombi ${ }^{20}$, A.J. Zsigmond

Institute of Nuclear Research ATOMKI, Debrecen, Hungary

N. Beni, S. Czellar, J. Molnar, J. Palinkas, Z. Szillasi

University of Debrecen, Debrecen, Hungary

J. Karancsi, P. Raics, Z.L. Trocsanyi, B. Ujvari

Panjab University, Chandigarh, India

S.B. Beri, V. Bhatnagar, N. Dhingra, R. Gupta, M. Kaur, M.Z. Mehta, M. Mittal, N. Nishu, L.K. Saini, A. Sharma, J.B. Singh

University of Delhi, Delhi, India

Ashok Kumar, Arun Kumar, S. Ahuja, A. Bhardwaj, B.C. Choudhary, S. Malhotra, M. Naimuddin, K. Ranjan, P. Saxena, V. Sharma, R.K. Shivpuri

Saha Institute of Nuclear Physics, Kolkata, India

S. Banerjee, S. Bhattacharya, K. Chatterjee, S. Dutta, B. Gomber, Sa. Jain, Sh. Jain, R. Khurana, A. Modak, S. Mukherjee, D. Roy, S. Sarkar, M. Sharan

Bhabha Atomic Research Centre, Mumbai, India

A. Abdulsalam, D. Dutta, S. Kailas, V. Kumar, A.K. Mohanty², L.M. Pant, P. Shukla, A. Topkar

Tata Institute of Fundamental Research - EHEP, Mumbai, India

T. Aziz, R.M. Chatterjee, S. Ganguly, M. Guchait ${ }^{21}$, A. Gurtu ${ }^{22}$, M. Maity ${ }^{23}$, G. Majumder, K. Mazumdar, G.B. Mohanty, B. Parida, K. Sudhakar, N. Wickramage

Tata Institute of Fundamental Research - HECR, Mumbai, India

S. Banerjee, S. Dugad

Institute for Research in Fundamental Sciences (IPM), Tehran, Iran

H. Arfaei ${ }^{24}$, H. Bakhshiansohi, S.M. Etesami ${ }^{25}$, A. Fahim ${ }^{24}$, H. Hesari, A. Jafari, M. Khakzad, M. Mohammadi Najafabadi, S. Paktinat Mehdiabadi, B. Safarzadeh ${ }^{26}$, M. Zeinali

University College Dublin, Dublin, Ireland

M. Grunewald

INFN Sezione di Bari ${ }^{a}$, Università di Bari ${ }^{b}$, Politecnico di Bari ${ }^{c}$, Bari, Italy M. Abbrescia $^{a, b}$, L. Barbone ${ }^{a, b}$, C. Calabria ${ }^{a, b, 2}$, S.S. Chhibra ${ }^{a, b}$, A. Colaleo ${ }^{a}$, D. Creanza ${ }^{a, c}$, N. De Filippis ${ }^{a, c, 2}$, M. De Palma ${ }^{a, b}$, L. Fiore ${ }^{a}$, G. Iaselli ${ }^{a, c}$, G. Maggi $^{a, c}$, M. Maggi ${ }^{a}$, B. Marangelli ${ }^{a, b}$, S. My ${ }^{a, c}$, S. $\mathrm{Nuzzo}^{a, b}$, N. Pacifico ${ }^{a}$, A. Pompili ${ }^{a, b}$,

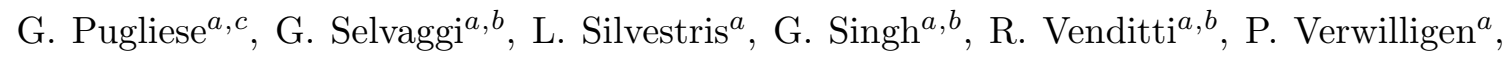
G. Zito ${ }^{a}$ 
INFN Sezione di Bologna ${ }^{a}$, Università di Bologna ${ }^{b}$, Bologna, Italy

G. Abbiendi $^{a}$, A.C. Benvenuti ${ }^{a}$, D. Bonacorsi ${ }^{a}, b$, S. Braibant-Giacomelli ${ }^{a, b}$,

L. Brigliadori ${ }^{a}, b$, R. Campanini ${ }^{a, b}$, P. Capiluppi ${ }^{a}, b$, A. Castro ${ }^{a}, b$ F.R. Cavallo ${ }^{a}$,

M. Cuffiani ${ }^{a, b}$, G.M. Dallavalle ${ }^{a}$, F. Fabbri ${ }^{a}$, A. Fanfani ${ }^{a, b}$, D. Fasanella ${ }^{a, b}$, P. Giacomelli $^{a}$,

C. Grandi ${ }^{a}$, L. Guiducci ${ }^{a}, b$, S. Marcellini ${ }^{a}$, G. Masetti ${ }^{a}$, M. Meneghelli ${ }^{a}, b, 2$, A. Montanari ${ }^{a}$,

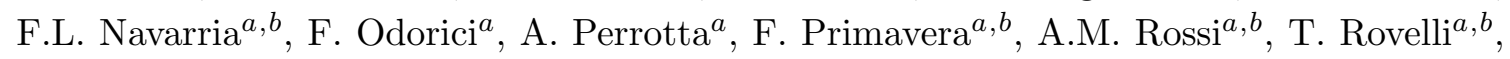
G.P. Siroli ${ }^{a, b}$, N. Tosi ${ }^{a, b}$, R. Travaglini ${ }^{a, b}$

INFN Sezione di Catania ${ }^{a}$, Università di Catania ${ }^{b}$, Catania, Italy

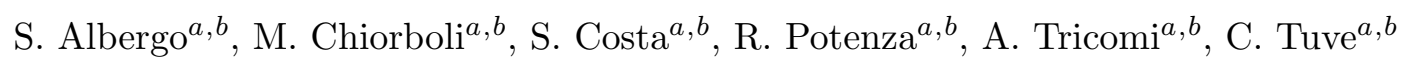

INFN Sezione di Firenze ${ }^{a}$, Università di Firenze ${ }^{b}$, Firenze, Italy

G. Barbagli ${ }^{a}$, V. Ciulli ${ }^{a}, b$, C. Civinini ${ }^{a}$, R. D’Alessandro ${ }^{a, b}$, E. Focardi ${ }^{a}, b$, S. Frosali ${ }^{a, b}$,

E. Gallo ${ }^{a}$, S. Gonzi ${ }^{a, b}$, P. Lenzi ${ }^{a, b}$, M. Meschini ${ }^{a}$, S. Paoletti ${ }^{a}$, G. Sguazzoni ${ }^{a}$,

A. Tropiano ${ }^{a, b}$

INFN Laboratori Nazionali di Frascati, Frascati, Italy

L. Benussi, S. Bianco, F. Fabbri, D. Piccolo

INFN Sezione di Genova ${ }^{a}$, Università di Genova ${ }^{b}$, Genova, Italy

P. Fabbricatore ${ }^{a}$, R. Musenich ${ }^{a}$, S. Tosi ${ }^{a, b}$

INFN Sezione di Milano-Bicocca ${ }^{a}$, Università di Milano-Bicocca ${ }^{b}$, Milano, Italy

A. Benaglia $^{a}$, F. De Guio ${ }^{a, b}$, L. Di Matteo ${ }^{a, b, 2}$, S. Fiorendi ${ }^{a, b}$, S. Gennai ${ }^{a, 2}$, A. Ghezzi $^{a, b}$,

P. Govoni, M.T. Lucchini ${ }^{2}$, S. Malvezzi ${ }^{a}$, R.A. Manzoni ${ }^{a, b}$, A. Martelli ${ }^{a}, b$, A. Massironi ${ }^{a, b}$,

D. Menasce ${ }^{a}$, L. Moroni $^{a}$, M. Paganoni ${ }^{a, b}$, D. Pedrini ${ }^{a}$, S. Ragazzi ${ }^{a, b}$, N. Redaelli ${ }^{a}$,

T. Tabarelli de Fatis ${ }^{a, b}$

INFN Sezione di Napoli ${ }^{a}$, Università di Napoli 'Federico II' ${ }^{b}$, Università della Basilicata (Potenza) ${ }^{c}$, Università G. Marconi (Roma) ${ }^{d}$, Napoli, Italy

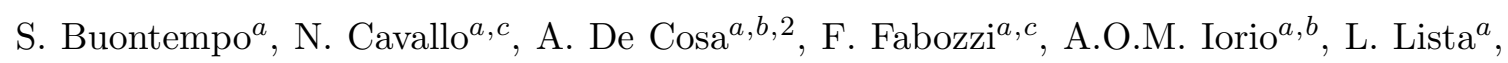
S. Meola ${ }^{a, d, 2}$, M. Merola ${ }^{a}$, P. Paolucci ${ }^{a, 2}$

INFN Sezione di Padova ${ }^{a}$, Università di Padova ${ }^{b}$, Università di Trento (Trento) ${ }^{c}$, Padova, Italy

P. Azzi ${ }^{a}$, N. Bacchetta ${ }^{a, 2}$, P. Bellan ${ }^{a, b}$, D. Bisello ${ }^{a, b}$, A. Branca ${ }^{a, b}$, R. Carlin ${ }^{a, b}$,

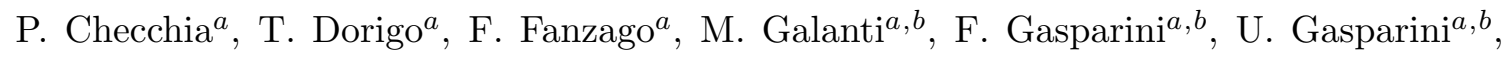
P. Giubilato ${ }^{a, b}$, F. Gonella ${ }^{a}$, A. Gozzelino ${ }^{a}$, M. Gulmini ${ }^{a, 27}$, K. Kanishchev ${ }^{a, c}$, S. Lacaprara ${ }^{a}$, I. Lazzizzera ${ }^{a, c}$, M. Margoni ${ }^{a, b}$, A.T. Meneguzzo ${ }^{a, b}$, J. Pazzini ${ }^{a, b}$, N. Pozzobon ${ }^{a, b}$, P. Ronchese ${ }^{a, b}$, F. Simonetto ${ }^{a, b}$, E. Torassa ${ }^{a}$, M. Tosi $^{a, b}$, P. Zotto $^{a, b}$, A. Zucchetta ${ }^{a, b}$, G. Zumerle ${ }^{a, b}$

INFN Sezione di Pavia ${ }^{a}$, Università di Pavia ${ }^{b}$, Pavia, Italy

M. Gabusi ${ }^{a, b}$, S.P. Ratti ${ }^{a, b}$, C. Riccardi ${ }^{a, b}$, P. Vitulo ${ }^{a, b}$ 
INFN Sezione di Perugia ${ }^{a}$, Università di Perugia ${ }^{b}$, Perugia, Italy

M. Biasini ${ }^{a}, b$, G.M. Bilei ${ }^{a}$, L. Fanò ${ }^{a, b}$, P. Lariccia ${ }^{a, b}$, G. Mantovani ${ }^{a}, b$, M. Menichelli ${ }^{a}$, A. Nappi ${ }^{a, b \dagger}$, F. Romeo ${ }^{a, b}$, A. Saha ${ }^{a}$, A. Santocchia ${ }^{a, b}$, A. Spiezia ${ }^{a, b}$

INFN Sezione di Pisa ${ }^{a}$, Università di Pisa ${ }^{b}$, Scuola Normale Superiore di Pisa ${ }^{c}$, Pisa, Italy

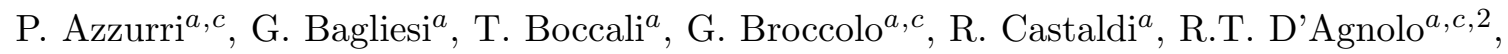

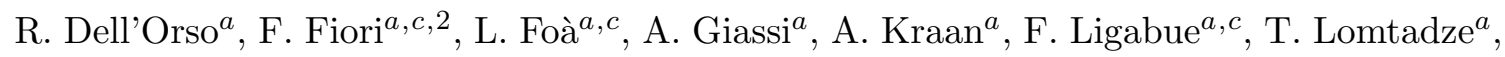
L. Martini ${ }^{a, 28}$, A. Messineo ${ }^{a, b}$, F. Palla ${ }^{a}$, A. Rizzi ${ }^{a}, b$ A.T. $\operatorname{Serban}^{a}$, P. Spagnolo ${ }^{a}$,

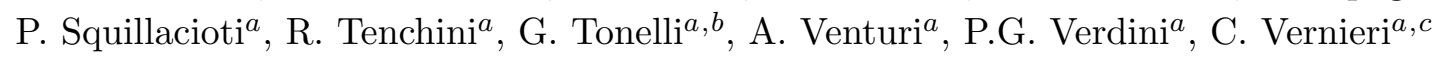

INFN Sezione di Roma ${ }^{a}$, Università di Roma ${ }^{b}$, Roma, Italy

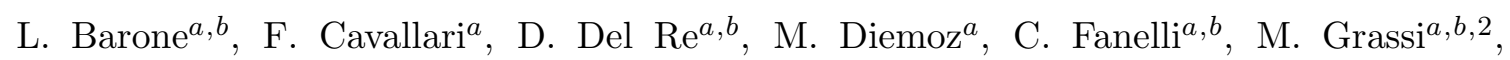
E. Longo ${ }^{a, b}$, F. Margaroli ${ }^{a, b}$, P. Meridiani ${ }^{a, 2}$, F. Micheli ${ }^{a, b}$, S. Nourbakhsh ${ }^{a, b}$, G. Organtini ${ }^{a, b}$, R. Paramatti ${ }^{a}$, S. Rahatlou ${ }^{a, b}$, L. Soffi ${ }^{a, b}$

INFN Sezione di Torino ${ }^{a}$, Università di Torino ${ }^{b}$, Università del Piemonte Orientale (Novara) ${ }^{c}$, Torino, Italy

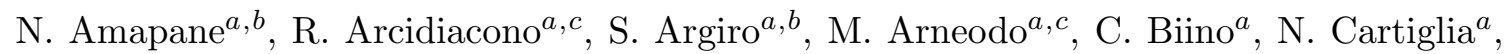

S. Casasso ${ }^{a, b}$, M. $\operatorname{Costa}^{a, b}$, N. Demaria ${ }^{a}$, C. Mariotti ${ }^{a, 2}$, S. Maselli ${ }^{a}$, G. Mazza ${ }^{a}$,

E. Migliore ${ }^{a, b}$, V. Monaco ${ }^{a, b}$, M. Musich ${ }^{a, 2}$, M.M. Obertino ${ }^{a, c}$, N. Pastrone ${ }^{a}$,

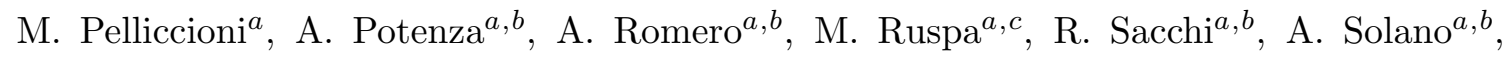
A. Staiano ${ }^{a}$, U. Tamponi ${ }^{a}$

INFN Sezione di Trieste ${ }^{a}$, Università di Trieste ${ }^{b}$, Trieste, Italy

S. Belforte ${ }^{a}$, V. Candelise ${ }^{a, b}$, M. Casarsa ${ }^{a}$, F. Cossutti ${ }^{a, 2}$, G. Della Ricca ${ }^{a, b}$, B. Gobbo ${ }^{a}$,

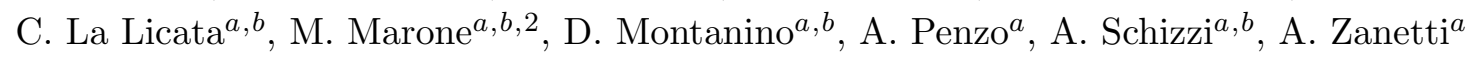

Kangwon National University, Chunchon, Korea

T.Y. Kim, S.K. Nam

Kyungpook National University, Daegu, Korea

S. Chang, D.H. Kim, G.N. Kim, J.E. Kim, D.J. Kong, Y.D. Oh, H. Park, D.C. Son

Chonnam National University, Institute for Universe and Elementary Particles, Kwangju, Korea

J.Y. Kim, Zero J. Kim, S. Song

Korea University, Seoul, Korea

S. Choi, D. Gyun, B. Hong, M. Jo, H. Kim, T.J. Kim, K.S. Lee, D.H. Moon, S.K. Park, Y. Roh

University of Seoul, Seoul, Korea

M. Choi, J.H. Kim, C. Park, I.C. Park, S. Park, G. Ryu

Sungkyunkwan University, Suwon, Korea

Y. Choi, Y.K. Choi, J. Goh, M.S. Kim, E. Kwon, B. Lee, J. Lee, S. Lee, H. Seo, I. Yu 
Vilnius University, Vilnius, Lithuania

I. Grigelionis, A. Juodagalvis

Centro de Investigacion y de Estudios Avanzados del IPN, Mexico City, Mexico

H. Castilla-Valdez, E. De La Cruz-Burelo, I. Heredia-de La Cruz, R. Lopez-Fernandez, J. Martínez-Ortega, A. Sanchez-Hernandez, L.M. Villasenor-Cendejas

Universidad Iberoamericana, Mexico City, Mexico

S. Carrillo Moreno, F. Vazquez Valencia

Benemerita Universidad Autonoma de Puebla, Puebla, Mexico

H.A. Salazar Ibarguen

Universidad Autónoma de San Luis Potosí, San Luis Potosí, Mexico

E. Casimiro Linares, A. Morelos Pineda, M.A. Reyes-Santos

University of Auckland, Auckland, New Zealand

D. Krofcheck

University of Canterbury, Christchurch, New Zealand

A.J. Bell, P.H. Butler, R. Doesburg, S. Reucroft, H. Silverwood

National Centre for Physics, Quaid-I-Azam University, Islamabad, Pakistan

M. Ahmad, M.I. Asghar, J. Butt, H.R. Hoorani, S. Khalid, W.A. Khan, T. Khurshid, S. Qazi, M.A. Shah, M. Shoaib

National Centre for Nuclear Research, Swierk, Poland

H. Bialkowska, B. Boimska, T. Frueboes, M. Górski, M. Kazana, K. Nawrocki, K. Romanowska-Rybinska, M. Szleper, G. Wrochna, P. Zalewski

Institute of Experimental Physics, Faculty of Physics, University of Warsaw, Warsaw, Poland

G. Brona, K. Bunkowski, M. Cwiok, W. Dominik, K. Doroba, A. Kalinowski, M. Konecki, J. Krolikowski, M. Misiura, W. Wolszczak

Laboratório de Instrumentação e Física Experimental de Partículas, Lisboa, Portugal

N. Almeida, P. Bargassa, A. David, P. Faccioli, P.G. Ferreira Parracho, M. Gallinaro, J. Seixas ${ }^{2}$, J. Varela, P. Vischia

Joint Institute for Nuclear Research, Dubna, Russia

P. Bunin, M. Gavrilenko, I. Golutvin, I. Gorbunov, A. Kamenev, V. Karjavin, V. Konoplyanikov, G. Kozlov, A. Lanev, A. Malakhov, P. Moisenz, V. Palichik, V. Perelygin, S. Shmatov, V. Smirnov, A. Volodko, A. Zarubin

Petersburg Nuclear Physics Institute, Gatchina (St. Petersburg), Russia

S. Evstyukhin, V. Golovtsov, Y. Ivanov, V. Kim, P. Levchenko, V. Murzin, V. Oreshkin, I. Smirnov, V. Sulimov, L. Uvarov, S. Vavilov, A. Vorobyev, An. Vorobyev 
Institute for Nuclear Research, Moscow, Russia

Yu. Andreev, A. Dermenev, S. Gninenko, N. Golubev, M. Kirsanov, N. Krasnikov, V. Matveev, A. Pashenkov, D. Tlisov, A. Toropin

Institute for Theoretical and Experimental Physics, Moscow, Russia

V. Epshteyn, M. Erofeeva, V. Gavrilov, N. Lychkovskaya, V. Popov, G. Safronov, S. Semenov, A. Spiridonov, V. Stolin, M. Toms, E. Vlasov, A. Zhokin

P.N. Lebedev Physical Institute, Moscow, Russia

V. Andreev, M. Azarkin, I. Dremin, M. Kirakosyan, A. Leonidov, G. Mesyats, S.V. Rusakov, A. Vinogradov

Skobeltsyn Institute of Nuclear Physics, Lomonosov Moscow State University, Moscow, Russia

A. Belyaev, E. Boos, M. Dubinin ${ }^{6}$, A. Ershov, L. Khein, V. Klyukhin, O. Kodolova,

I. Lokhtin, A. Markina, S. Obraztsov, S. Petrushanko, A. Proskuryakov, V. Savrin,

A. Snigirev

State Research Center of Russian Federation, Institute for High Energy Physics, Protvino, Russia

I. Azhgirey, I. Bayshev, S. Bitioukov, V. Kachanov, A. Kalinin, D. Konstantinov,

V. Krychkine, V. Petrov, R. Ryutin, A. Sobol, L. Tourtchanovitch, S. Troshin, N. Tyurin,

A. Uzunian, A. Volkov

University of Belgrade, Faculty of Physics and Vinca Institute of Nuclear Sciences, Belgrade, Serbia

P. Adzic ${ }^{29}$, M. Ekmedzic, D. Krpic ${ }^{29}$, J. Milosevic

Centro de Investigaciones Energéticas Medioambientales y Tecnológicas (CIEMAT), Madrid, Spain

M. Aguilar-Benitez, J. Alcaraz Maestre, C. Battilana, E. Calvo, M. Cerrada, M. Chamizo Llatas $^{2}$, N. Colino, B. De La Cruz, A. Delgado Peris, D. Domínguez Vázquez, C. Fernandez Bedoya, J.P. Fernández Ramos, A. Ferrando, J. Flix, M.C. Fouz, P. Garcia-Abia, O. Gonzalez Lopez, S. Goy Lopez, J.M. Hernandez, M.I. Josa, G. Merino, E. Navarro De Martino, J. Puerta Pelayo, A. Quintario Olmeda, I. Redondo, L. Romero, J. Santaolalla, M.S. Soares, C. Willmott

Universidad Autónoma de Madrid, Madrid, Spain

C. Albajar, J.F. de Trocóniz

Universidad de Oviedo, Oviedo, Spain

H. Brun, J. Cuevas, J. Fernandez Menendez, S. Folgueras, I. Gonzalez Caballero, L. Lloret Iglesias, J. Piedra Gomez

Instituto de Física de Cantabria (IFCA), CSIC-Universidad de Cantabria, Santander, Spain

J.A. Brochero Cifuentes, I.J. Cabrillo, A. Calderon, S.H. Chuang, J. Duarte Campderros, M. Fernandez, G. Gomez, J. Gonzalez Sanchez, A. Graziano, C. Jorda, A. Lopez Virto, 
J. Marco, R. Marco, C. Martinez Rivero, F. Matorras, F.J. Munoz Sanchez, T. Rodrigo, A.Y. Rodríguez-Marrero, A. Ruiz-Jimeno, L. Scodellaro, I. Vila, R. Vilar Cortabitarte

\section{CERN, European Organization for Nuclear Research, Geneva, Switzerland}

D. Abbaneo, E. Auffray, G. Auzinger, M. Bachtis, P. Baillon, A.H. Ball, D. Barney, J. Bendavid, J.F. Benitez, C. Bernet ${ }^{7}$, G. Bianchi, P. Bloch, A. Bocci, A. Bonato, C. Botta, H. Breuker, T. Camporesi, G. Cerminara, T. Christiansen, J.A. Coarasa Perez, S. Colafranceschi ${ }^{30}$, D. d'Enterria, A. Dabrowski, A. De Roeck, S. De Visscher, S. Di Guida, M. Dobson, N. Dupont-Sagorin, A. Elliott-Peisert, J. Eugster, W. Funk, G. Georgiou, M. Giffels, D. Gigi, K. Gill, D. Giordano, M. Girone, M. Giunta, F. Glege, R. GomezReino Garrido, S. Gowdy, R. Guida, J. Hammer, M. Hansen, P. Harris, C. Hartl, B. Hegner, A. Hinzmann, V. Innocente, P. Janot, K. Kaadze, E. Karavakis, K. Kousouris, K. Krajczar, P. Lecoq, Y.-J. Lee, C. Lourenço, N. Magini, M. Malberti, L. Malgeri, M. Mannelli, L. Masetti, F. Meijers, S. Mersi, E. Meschi, R. Moser, M. Mulders, P. Musella, E. Nesvold, L. Orsini, E. Palencia Cortezon, E. Perez, L. Perrozzi, A. Petrilli, A. Pfeiffer, M. Pierini, M. Pimiä, D. Piparo, G. Polese, L. Quertenmont, A. Racz, W. Reece, J. Rodrigues Antunes, G. Rolandi ${ }^{31}$, C. Rovelli ${ }^{32}$, M. Rovere, H. Sakulin, F. Santanastasio, C. Schäfer, C. Schwick, I. Segoni, S. Sekmen, A. Sharma, P. Siegrist, P. Silva, M. Simon, P. Sphicas ${ }^{33}$, D. Spiga, M. Stoye, A. Tsirou, G.I. Veres ${ }^{20}$, J.R. Vlimant, H.K. Wöhri, S.D. Worm ${ }^{34}$, W.D. Zeuner

\section{Paul Scherrer Institut, Villigen, Switzerland}

W. Bertl, K. Deiters, W. Erdmann, K. Gabathuler, R. Horisberger, Q. Ingram, H.C. Kaestli, S. König, D. Kotlinski, U. Langenegger, F. Meier, D. Renker, T. Rohe

\section{Institute for Particle Physics, ETH Zurich, Zurich, Switzerland}

F. Bachmair, L. Bäni, P. Bortignon, M.A. Buchmann, B. Casal, N. Chanon, A. Deisher, G. Dissertori, M. Dittmar, M. Donegà, M. Dünser, P. Eller, C. Grab, D. Hits, P. Lecomte, W. Lustermann, A.C. Marini, P. Martinez Ruiz del Arbol, N. Mohr, F. Moortgat, C. Nägeli ${ }^{35}$, P. Nef, F. Nessi-Tedaldi, F. Pandolfi, L. Pape, F. Pauss, M. Peruzzi, F.J. Ronga, M. Rossini, L. Sala, A.K. Sanchez, A. Starodumov ${ }^{36}$, B. Stieger, M. Takahashi, L. Tauscher ${ }^{\dagger}$, A. Thea, K. Theofilatos, D. Treille, C. Urscheler, R. Wallny, H.A. Weber

\section{Universität Zürich, Zurich, Switzerland}

C. Amsler ${ }^{37}$, V. Chiochia, C. Favaro, M. Ivova Rikova, B. Kilminster, B. Millan Mejias, P. Otiougova, P. Robmann, H. Snoek, S. Taroni, S. Tupputi, M. Verzetti

\section{National Central University, Chung-Li, Taiwan}

M. Cardaci, K.H. Chen, C. Ferro, C.M. Kuo, S.W. Li, W. Lin, Y.J. Lu, R. Volpe, S.S. Yu

\section{National Taiwan University (NTU), Taipei, Taiwan}

P. Bartalini, P. Chang, Y.H. Chang, Y.W. Chang, Y. Chao, K.F. Chen, C. Dietz, U. Grundler, W.-S. Hou, Y. Hsiung, K.Y. Kao, Y.J. Lei, R.-S. Lu, D. Majumder, E. Petrakou, X. Shi, J.G. Shiu, Y.M. Tzeng, M. Wang

\section{Chulalongkorn University, Bangkok, Thailand}

B. Asavapibhop, N. Suwonjandee 
Cukurova University, Adana, Turkey

A. Adiguzel, M.N. Bakirci ${ }^{38}$, S. Cerci ${ }^{39}$, C. Dozen, I. Dumanoglu, E. Eskut, S. Girgis, G. Gokbulut, E. Gurpinar, I. Hos, E.E. Kangal, A. Kayis Topaksu, G. Onengut, K. Ozdemir, S. Ozturk ${ }^{40}$, A. Polatoz, K. Sogut ${ }^{41}$, D. Sunar Cerci ${ }^{39}$, B. Tali ${ }^{39}$, H. Topakli ${ }^{38}$, M. Vergili

\section{Middle East Technical University, Physics Department, Ankara, Turkey}

I.V. Akin, T. Aliev, B. Bilin, S. Bilmis, M. Deniz, H. Gamsizkan, A.M. Guler, G. Karapinar ${ }^{42}$, K. Ocalan, A. Ozpineci, M. Serin, R. Sever, U.E. Surat, M. Yalvac, M. Zeyrek

\section{Bogazici University, Istanbul, Turkey}

E. Gülmez, B. Isildak ${ }^{43}$, M. Kaya ${ }^{44}$, O. Kaya ${ }^{44}$, S. Ozkorucuklu ${ }^{45}$, N. Sonmez ${ }^{46}$

Istanbul Technical University, Istanbul, Turkey

H. Bahtiyar ${ }^{47}$, E. Barlas, K. Cankocak, Y.O. Günaydin ${ }^{48}$, F.I. Vardarlı, M. Yücel

National Scientific Center, Kharkov Institute of Physics and Technology, Kharkov, Ukraine

L. Levchuk, P. Sorokin

University of Bristol, Bristol, United Kingdom

J.J. Brooke, E. Clement, D. Cussans, H. Flacher, R. Frazier, J. Goldstein, M. Grimes, G.P. Heath, H.F. Heath, L. Kreczko, S. Metson, D.M. Newbold ${ }^{34}$, K. Nirunpong, A. Poll, S. Senkin, V.J. Smith, T. Williams

Rutherford Appleton Laboratory, Didcot, United Kingdom

L. Basso ${ }^{49}$, K.W. Bell, A. Belyaev ${ }^{49}$, C. Brew, R.M. Brown, D.J.A. Cockerill, J.A. Coughlan, K. Harder, S. Harper, J. Jackson, E. Olaiya, D. Petyt, B.C. Radburn-Smith, C.H. Shepherd-Themistocleous, I.R. Tomalin, W.J. Womersley

Imperial College, London, United Kingdom

R. Bainbridge, G. Ball, O. Buchmuller, D. Burton, D. Colling, N. Cripps, M. Cutajar, P. Dauncey, G. Davies, M. Della Negra, W. Ferguson, J. Fulcher, D. Futyan, A. Gilbert, A. Guneratne Bryer, G. Hall, Z. Hatherell, J. Hays, G. Iles, M. Jarvis, G. Karapostoli, M. Kenzie, R. Lane, R. Lucas, L. Lyons, A.-M. Magnan, J. Marrouche, B. Mathias, R. Nandi, J. Nash, A. Nikitenko ${ }^{36}$, J. Pela, M. Pesaresi, K. Petridis, M. Pioppi ${ }^{50}$, D.M. Raymond, S. Rogerson, A. Rose, C. Seez, P. Sharp ${ }^{\dagger}$, A. Sparrow, A. Tapper, M. Vazquez Acosta, T. Virdee, S. Wakefield, N. Wardle, T. Whyntie

Brunel University, Uxbridge, United Kingdom

M. Chadwick, J.E. Cole, P.R. Hobson, A. Khan, P. Kyberd, D. Leggat, D. Leslie, W. Martin, I.D. Reid, P. Symonds, L. Teodorescu, M. Turner

\section{Baylor University, Waco, USA}

J. Dittmann, K. Hatakeyama, A. Kasmi, H. Liu, T. Scarborough

The University of Alabama, Tuscaloosa, USA

O. Charaf, S.I. Cooper, C. Henderson, P. Rumerio 


\section{Boston University, Boston, USA}

A. Avetisyan, T. Bose, C. Fantasia, A. Heister, P. Lawson, D. Lazic, J. Rohlf, D. Sperka, J. St. John, L. Sulak

\section{Brown University, Providence, USA}

J. Alimena, S. Bhattacharya, G. Christopher, D. Cutts, Z. Demiragli, A. Ferapontov, A. Garabedian, U. Heintz, G. Kukartsev, E. Laird, G. Landsberg, M. Luk, M. Narain, M. Segala, T. Sinthuprasith, T. Speer

\section{University of California, Davis, Davis, USA}

R. Breedon, G. Breto, M. Calderon De La Barca Sanchez, S. Chauhan, M. Chertok, J. Conway, R. Conway, P.T. Cox, R. Erbacher, M. Gardner, R. Houtz, W. Ko, A. Kopecky, R. Lander, O. Mall, T. Miceli, R. Nelson, D. Pellett, F. Ricci-Tam, B. Rutherford, M. Searle, J. Smith, M. Squires, M. Tripathi, R. Yohay

University of California, Los Angeles, USA

V. Andreev, D. Cline, R. Cousins, S. Erhan, P. Everaerts, C. Farrell, M. Felcini, J. Hauser, M. Ignatenko, C. Jarvis, G. Rakness, P. Schlein ${ }^{\dagger}$, P. Traczyk, V. Valuev, M. Weber

University of California, Riverside, Riverside, USA

J. Babb, R. Clare, M.E. Dinardo, J. Ellison, J.W. Gary, F. Giordano, G. Hanson, H. Liu, O.R. Long, A. Luthra, H. Nguyen, S. Paramesvaran, J. Sturdy, S. Sumowidagdo, R. Wilken, S. Wimpenny

\section{University of California, San Diego, La Jolla, USA}

W. Andrews, J.G. Branson, G.B. Cerati, S. Cittolin, D. Evans, A. Holzner, R. Kelley, M. Lebourgeois, J. Letts, I. Macneill, B. Mangano, S. Padhi, C. Palmer, G. Petrucciani, M. Pieri, M. Sani, V. Sharma, S. Simon, E. Sudano, M. Tadel, Y. Tu, A. Vartak, S. Wasserbaech ${ }^{51}$, F. Würthwein, A. Yagil, J. Yoo

\section{University of California, Santa Barbara, Santa Barbara, USA}

D. Barge, R. Bellan, C. Campagnari, M. D’Alfonso, T. Danielson, K. Flowers, P. Geffert, C. George, F. Golf, J. Incandela, C. Justus, P. Kalavase, D. Kovalskyi, V. Krutelyov, S. Lowette, R. Magaña Villalba, N. Mccoll, V. Pavlunin, J. Ribnik, J. Richman, R. Rossin, D. Stuart, W. To, C. West

\section{California Institute of Technology, Pasadena, USA}

A. Apresyan, A. Bornheim, J. Bunn, Y. Chen, E. Di Marco, J. Duarte, D. Kcira, Y. Ma, A. Mott, H.B. Newman, C. Rogan, M. Spiropulu, V. Timciuc, J. Veverka, R. Wilkinson, S. Xie, Y. Yang, R.Y. Zhu

\section{Carnegie Mellon University, Pittsburgh, USA}

V. Azzolini, A. Calamba, R. Carroll, T. Ferguson, Y. Iiyama, D.W. Jang, Y.F. Liu, M. Paulini, J. Russ, H. Vogel, I. Vorobiev

\section{University of Colorado at Boulder, Boulder, USA}

J.P. Cumalat, B.R. Drell, W.T. Ford, A. Gaz, E. Luiggi Lopez, U. Nauenberg, J.G. Smith, K. Stenson, K.A. Ulmer, S.R. Wagner 


\section{Cornell University, Ithaca, USA}

J. Alexander, A. Chatterjee, N. Eggert, L.K. Gibbons, W. Hopkins, A. Khukhunaishvili, B. Kreis, N. Mirman, G. Nicolas Kaufman, J.R. Patterson, A. Ryd, E. Salvati, W. Sun, W.D. Teo, J. Thom, J. Thompson, J. Tucker, Y. Weng, L. Winstrom, P. Wittich

\section{Fairfield University, Fairfield, USA}

D. Winn

\section{Fermi National Accelerator Laboratory, Batavia, USA}

S. Abdullin, M. Albrow, J. Anderson, G. Apollinari, L.A.T. Bauerdick, A. Beretvas, J. Berryhill, P.C. Bhat, K. Burkett, J.N. Butler, V. Chetluru, H.W.K. Cheung, F. Chlebana, S. Cihangir, V.D. Elvira, I. Fisk, J. Freeman, Y. Gao, E. Gottschalk, L. Gray, D. Green, O. Gutsche, R.M. Harris, J. Hirschauer, B. Hooberman, S. Jindariani, M. Johnson, U. Joshi, B. Klima, S. Kunori, S. Kwan, J. Linacre, D. Lincoln, R. Lipton, J. Lykken, K. Maeshima, J.M. Marraffino, V.I. Martinez Outschoorn, S. Maruyama, D. Mason, P. McBride, K. Mishra, S. Mrenna, Y. Musienko ${ }^{52}$, C. Newman-Holmes, V. O'Dell, O. Prokofyev, E. Sexton-Kennedy, S. Sharma, W.J. Spalding, L. Spiegel, L. Taylor, S. Tkaczyk, N.V. Tran, L. Uplegger, E.W. Vaandering, R. Vidal, J. Whitmore, W. Wu, F. Yang, J.C. Yun

\section{University of Florida, Gainesville, USA}

D. Acosta, P. Avery, D. Bourilkov, M. Chen, T. Cheng, S. Das, M. De Gruttola, G.P. Di Giovanni, D. Dobur, A. Drozdetskiy, R.D. Field, M. Fisher, Y. Fu, I.K. Furic, J. Hugon, B. Kim, J. Konigsberg, A. Korytov, A. Kropivnitskaya, T. Kypreos, J.F. Low, K. Matchev, P. Milenovic ${ }^{53}$, G. Mitselmakher, L. Muniz, R. Remington, A. Rinkevicius, N. Skhirtladze, M. Snowball, J. Yelton, M. Zakaria

\section{Florida International University, Miami, USA}

V. Gaultney, S. Hewamanage, L.M. Lebolo, S. Linn, P. Markowitz, G. Martinez, J.L. Rodriguez

\section{Florida State University, Tallahassee, USA}

T. Adams, A. Askew, J. Bochenek, J. Chen, B. Diamond, S.V. Gleyzer, J. Haas, S. Hagopian, V. Hagopian, K.F. Johnson, H. Prosper, V. Veeraraghavan, M. Weinberg

\section{Florida Institute of Technology, Melbourne, USA}

M.M. Baarmand, B. Dorney, M. Hohlmann, H. Kalakhety, F. Yumiceva

\section{University of Illinois at Chicago (UIC), Chicago, USA}

M.R. Adams, L. Apanasevich, V.E. Bazterra, R.R. Betts, I. Bucinskaite, J. Callner, R. Cavanaugh, O. Evdokimov, L. Gauthier, C.E. Gerber, D.J. Hofman, S. Khalatyan, P. Kurt, F. Lacroix, C. O'Brien, C. Silkworth, D. Strom, P. Turner, N. Varelas

\section{The University of Iowa, Iowa City, USA}

U. Akgun, E.A. Albayrak, B. Bilki ${ }^{54}$, W. Clarida, K. Dilsiz, F. Duru, S. Griffiths, J.P. Merlo, H. Mermerkaya ${ }^{55}$, A. Mestvirishvili, A. Moeller, J. Nachtman, C.R. Newsom, H. Ogul, Y. Onel, F. Ozok ${ }^{47}$, S. Sen, P. Tan, E. Tiras, J. Wetzel, T. Yetkin ${ }^{56}$, K. Yi 
Johns Hopkins University, Baltimore, USA

B.A. Barnett, B. Blumenfeld, S. Bolognesi, D. Fehling, G. Giurgiu, A.V. Gritsan, G. Hu, P. Maksimovic, M. Swartz, A. Whitbeck

The University of Kansas, Lawrence, USA

P. Baringer, A. Bean, G. Benelli, R.P. Kenny III, M. Murray, D. Noonan, S. Sanders, R. Stringer, J.S. Wood

Kansas State University, Manhattan, USA

A.F. Barfuss, I. Chakaberia, A. Ivanov, S. Khalil, M. Makouski, Y. Maravin, S. Shrestha, I. Svintradze

Lawrence Livermore National Laboratory, Livermore, USA

J. Gronberg, D. Lange, F. Rebassoo, D. Wright

University of Maryland, College Park, USA

A. Baden, B. Calvert, S.C. Eno, J.A. Gomez, N.J. Hadley, R.G. Kellogg, T. Kolberg, Y. Lu, M. Marionneau, A.C. Mignerey, K. Pedro, A. Peterman, A. Skuja, J. Temple, M.B. Tonjes, S.C. Tonwar

\section{Massachusetts Institute of Technology, Cambridge, USA}

A. Apyan, G. Bauer, W. Busza, E. Butz, I.A. Cali, M. Chan, V. Dutta, G. Gomez Ceballos, M. Goncharov, Y. Kim, M. Klute, A. Levin, P.D. Luckey, T. Ma, S. Nahn, C. Paus, D. Ralph, C. Roland, G. Roland, G.S.F. Stephans, F. Stöckli, K. Sumorok, K. Sung, D. Velicanu, R. Wolf, B. Wyslouch, M. Yang, Y. Yilmaz, A.S. Yoon, M. Zanetti, V. Zhukova

University of Minnesota, Minneapolis, USA

B. Dahmes, A. De Benedetti, G. Franzoni, A. Gude, J. Haupt, S.C. Kao, K. Klapoetke, Y. Kubota, J. Mans, N. Pastika, R. Rusack, M. Sasseville, A. Singovsky, N. Tambe, J. Turkewitz

University of Mississippi, Oxford, USA

L.M. Cremaldi, R. Kroeger, L. Perera, R. Rahmat, D.A. Sanders, D. Summers

University of Nebraska-Lincoln, Lincoln, USA

E. Avdeeva, K. Bloom, S. Bose, D.R. Claes, A. Dominguez, M. Eads, R. Gonzalez Suarez, J. Keller, I. Kravchenko, J. Lazo-Flores, S. Malik, G.R. Snow

State University of New York at Buffalo, Buffalo, USA

J. Dolen, A. Godshalk, I. Iashvili, S. Jain, A. Kharchilava, A. Kumar, S. Rappoccio, Z. Wan Northeastern University, Boston, USA

G. Alverson, E. Barberis, D. Baumgartel, M. Chasco, J. Haley, D. Nash, T. Orimoto, D. Trocino, D. Wood, J. Zhang

Northwestern University, Evanston, USA

A. Anastassov, K.A. Hahn, A. Kubik, L. Lusito, N. Mucia, N. Odell, B. Pollack, A. Pozdnyakov, M. Schmitt, S. Stoynev, M. Velasco, S. Won 


\section{University of Notre Dame, Notre Dame, USA}

D. Berry, A. Brinkerhoff, K.M. Chan, M. Hildreth, C. Jessop, D.J. Karmgard, J. Kolb, K. Lannon, W. Luo, S. Lynch, N. Marinelli, D.M. Morse, T. Pearson, M. Planer, R. Ruchti, J. Slaunwhite, N. Valls, M. Wayne, M. Wolf

\section{The Ohio State University, Columbus, USA}

L. Antonelli, B. Bylsma, L.S. Durkin, C. Hill, R. Hughes, K. Kotov, T.Y. Ling, D. Puigh, M. Rodenburg, G. Smith, C. Vuosalo, G. Williams, B.L. Winer, H. Wolfe

\section{Princeton University, Princeton, USA}

E. Berry, P. Elmer, V. Halyo, P. Hebda, J. Hegeman, A. Hunt, P. Jindal, S.A. Koay, D. Lopes Pegna, P. Lujan, D. Marlow, T. Medvedeva, M. Mooney, J. Olsen, P. Piroué, X. Quan, A. Raval, H. Saka, D. Stickland, C. Tully, J.S. Werner, S.C. Zenz, A. Zuranski

\section{University of Puerto Rico, Mayaguez, USA}

E. Brownson, A. Lopez, H. Mendez, J.E. Ramirez Vargas

\section{Purdue University, West Lafayette, USA}

E. Alagoz, D. Benedetti, G. Bolla, D. Bortoletto, M. De Mattia, A. Everett, Z. Hu, M. Jones, O. Koybasi, M. Kress, N. Leonardo, V. Maroussov, P. Merkel, D.H. Miller, N. Neumeister, I. Shipsey, D. Silvers, A. Svyatkovskiy, M. Vidal Marono, H.D. Yoo, J. Zablocki, Y. Zheng

\section{Purdue University Calumet, Hammond, USA}

S. Guragain, N. Parashar

\section{Rice University, Houston, USA}

A. Adair, B. Akgun, K.M. Ecklund, F.J.M. Geurts, W. Li, B.P. Padley, R. Redjimi, J. Roberts, J. Zabel

\section{University of Rochester, Rochester, USA}

B. Betchart, A. Bodek, R. Covarelli, P. de Barbaro, R. Demina, Y. Eshaq, T. Ferbel, A. Garcia-Bellido, P. Goldenzweig, J. Han, A. Harel, D.C. Miner, G. Petrillo, D. Vishnevskiy, M. Zielinski

The Rockefeller University, New York, USA

A. Bhatti, R. Ciesielski, L. Demortier, K. Goulianos, G. Lungu, S. Malik, C. Mesropian

Rutgers, The State University of New Jersey, Piscataway, USA

S. Arora, A. Barker, J.P. Chou, C. Contreras-Campana, E. Contreras-Campana, D. Duggan, D. Ferencek, Y. Gershtein, R. Gray, E. Halkiadakis, D. Hidas, A. Lath, S. Panwalkar, M. Park, R. Patel, V. Rekovic, J. Robles, K. Rose, S. Salur, S. Schnetzer, C. Seitz, S. Somalwar, R. Stone, S. Thomas, M. Walker

University of Tennessee, Knoxville, USA

G. Cerizza, M. Hollingsworth, S. Spanier, Z.C. Yang, A. York 


\section{Texas A\&M University, College Station, USA}

R. Eusebi, W. Flanagan, J. Gilmore, T. Kamon ${ }^{57}$, V. Khotilovich, R. Montalvo, I. Osipenkov, Y. Pakhotin, A. Perloff, J. Roe, A. Safonov, T. Sakuma, I. Suarez, A. Tatarinov, D. Toback

\section{Texas Tech University, Lubbock, USA}

N. Akchurin, J. Damgov, C. Dragoiu, P.R. Dudero, C. Jeong, K. Kovitanggoon, S.W. Lee, T. Libeiro, I. Volobouev

\section{Vanderbilt University, Nashville, USA}

E. Appelt, A.G. Delannoy, S. Greene, A. Gurrola, W. Johns, C. Maguire, Y. Mao, A. Melo, M. Sharma, P. Sheldon, B. Snook, S. Tuo, J. Velkovska

\section{University of Virginia, Charlottesville, USA}

M.W. Arenton, M. Balazs, S. Boutle, B. Cox, B. Francis, J. Goodell, R. Hirosky, A. Ledovskoy, C. Lin, C. Neu, J. Wood

\section{Wayne State University, Detroit, USA}

S. Gollapinni, R. Harr, P.E. Karchin, C. Kottachchi Kankanamge Don, P. Lamichhane, A. Sakharov

\section{University of Wisconsin, Madison, USA}

M. Anderson, D.A. Belknap, L. Borrello, D. Carlsmith, M. Cepeda, S. Dasu, E. Friis, K.S. Grogg, M. Grothe, R. Hall-Wilton, M. Herndon, A. Hervé, P. Klabbers, J. Klukas, A. Lanaro, C. Lazaridis, R. Loveless, A. Mohapatra, M.U. Mozer, I. Ojalvo, G.A. Pierro, I. Ross, A. Savin, W.H. Smith, J. Swanson

\footnotetext{
$\dagger$ : Deceased

1: Also at Vienna University of Technology, Vienna, Austria

2: Also at CERN, European Organization for Nuclear Research, Geneva, Switzerland

3: Also at National Institute of Chemical Physics and Biophysics, Tallinn, Estonia

4: Also at Skobeltsyn Institute of Nuclear Physics, Lomonosov Moscow State University, Moscow, Russia

5: Also at Universidade Estadual de Campinas, Campinas, Brazil

6: Also at California Institute of Technology, Pasadena, USA

7: Also at Laboratoire Leprince-Ringuet, Ecole Polytechnique, IN2P3-CNRS, Palaiseau, France

8: Also at Suez Canal University, Suez, Egypt

9: Also at Cairo University, Cairo, Egypt

10: Also at Fayoum University, El-Fayoum, Egypt

11: Also at Helwan University, Cairo, Egypt

12: Also at British University in Egypt, Cairo, Egypt

13: Now at Ain Shams University, Cairo, Egypt

14: Also at National Centre for Nuclear Research, Swierk, Poland

15: Also at Université de Haute Alsace, Mulhouse, France

16: Also at Joint Institute for Nuclear Research, Dubna, Russia

17: Also at Brandenburg University of Technology, Cottbus, Germany
} 
18: Also at The University of Kansas, Lawrence, USA

19: Also at Institute of Nuclear Research ATOMKI, Debrecen, Hungary

20: Also at Eötvös Loránd University, Budapest, Hungary

21: Also at Tata Institute of Fundamental Research - HECR, Mumbai, India

22: Now at King Abdulaziz University, Jeddah, Saudi Arabia

23: Also at University of Visva-Bharati, Santiniketan, India

24: Also at Sharif University of Technology, Tehran, Iran

25: Also at Isfahan University of Technology, Isfahan, Iran

26: Also at Plasma Physics Research Center, Science and Research Branch, Islamic Azad University, Tehran, Iran

27: Also at Laboratori Nazionali di Legnaro dell' INFN, Legnaro, Italy

28: Also at Università degli Studi di Siena, Siena, Italy

29: Also at Faculty of Physics, University of Belgrade, Belgrade, Serbia

30: Also at Facoltà Ingegneria, Università di Roma, Roma, Italy

31: Also at Scuola Normale e Sezione dell'INFN, Pisa, Italy

32: Also at INFN Sezione di Roma, Roma, Italy

33: Also at University of Athens, Athens, Greece

34: Also at Rutherford Appleton Laboratory, Didcot, United Kingdom

35: Also at Paul Scherrer Institut, Villigen, Switzerland

36: Also at Institute for Theoretical and Experimental Physics, Moscow, Russia

37: Also at Albert Einstein Center for Fundamental Physics, Bern, Switzerland

38: Also at Gaziosmanpasa University, Tokat, Turkey

39: Also at Adiyaman University, Adiyaman, Turkey

40: Also at The University of Iowa, Iowa City, USA

41: Also at Mersin University, Mersin, Turkey

42: Also at Izmir Institute of Technology, Izmir, Turkey

43: Also at Ozyegin University, Istanbul, Turkey

44: Also at Kafkas University, Kars, Turkey

45: Also at Suleyman Demirel University, Isparta, Turkey

46: Also at Ege University, Izmir, Turkey

47: Also at Mimar Sinan University, Istanbul, Istanbul, Turkey

48: Also at Kahramanmaras Sütcü Imam University, Kahramanmaras, Turkey

49: Also at School of Physics and Astronomy, University of Southampton, Southampton, United Kingdom

50: Also at INFN Sezione di Perugia; Università di Perugia, Perugia, Italy

51: Also at Utah Valley University, Orem, USA

52: Also at Institute for Nuclear Research, Moscow, Russia

53: Also at University of Belgrade, Faculty of Physics and Vinca Institute of Nuclear Sciences, Belgrade, Serbia

54: Also at Argonne National Laboratory, Argonne, USA

55: Also at Erzincan University, Erzincan, Turkey

56: Also at Yildiz Technical University, Istanbul, Turkey

57: Also at Kyungpook National University, Daegu, Korea 